\title{
Sets of interpolation and sampling for weighted Banach spaces of holomorphic functions
}

\author{
by Pawee Domański (Poznań) and Mikael Lindström (Åbo)
}

\begin{abstract}
We give an elementary approach which allows us to evaluate Seip's conditions characterizing interpolating and sampling sequences in weighted Bergman spaces of infinite order for a wide class of weights depending on the distance to the boundary of the domain. Our results also give some information on cases not covered by Seip's theory. Moreover, we obtain new criteria for weights to be essential.
\end{abstract}

1. Introduction. We study sequences of interpolation and sampling in weighted Bergman spaces of infinite order:

$$
B_{v}^{\infty}:=\left\{f: \mathbb{D} \rightarrow \mathbb{C}: f \text { analytic, }\|f\|_{v}:=\sup _{z \in \mathbb{D}} v(z)|f(z)|<\infty\right\}
$$

endowed with the norm $\|\cdot\|_{v}$, where $v: \mathbb{D} \rightarrow \mathbb{R}_{+}$is an arbitrary weight, i.e., a continuous strictly positive function on the unit disc $\mathbb{D}$. By the Riemann Mapping Theorem, we can consider, in fact, weighted Bergman spaces $B_{v}^{\infty}(\Omega)$ on arbitrary simply connected domains $\Omega \subseteq \mathbb{C}, \Omega \neq \mathbb{C}$. We are especially interested in weights $v: \Omega \rightarrow \mathbb{R}_{+}$of the form $v(z)=\varphi(\operatorname{dist}(z, \partial \Omega))$.

For a sequence $\left(z_{n}\right) \subset \mathbb{D}$, let us define a restriction operator

$$
T: B_{v}^{\infty} \rightarrow l^{\infty}, \quad T(f)=\left(f\left(z_{n}\right) v\left(z_{n}\right)\right)_{n \in \mathbb{N}} .
$$

We call a sequence $\left(z_{n}\right)$ of distinct points in $\mathbb{D}$ a set of interpolation, of linear interpolation or of sampling for $v$, respectively, if $T$ is surjective, has a continuous linear right inverse or is a topological into isomorphism, respectively.

In a fundamental paper [S2] (cf. [HKZ, Section 5]) Seip characterized sets of interpolation and sampling in classical Bergman spaces with weights $v_{p}(z):=\left(1-|z|^{2}\right)^{p}, p>0$, on $\mathbb{D}$ as follows.

2000 Mathematics Subject Classification: Primary 30E05, 46E15.

Key words and phrases: weighted Bergman spaces of infinite order, interpolation, sampling, essential weight.

The research of the authors was partially supported by Komitet Badań Naukowych (State Committee for Scientific Research), Poland, grant no. 2 P03A 05115 and Academy of Finland, respectively. 
Theorem A. A sequence $\Gamma$ of distinct points in $\mathbb{D}$ is a set of sampling for $v_{p}$ if and only if it contains a uniformly discrete subsequence $\Gamma^{\prime}$ for which $D^{-}\left(\Gamma^{\prime}\right)>p$. Moreover, if $\Gamma$ is a uniformly discrete set of sampling for the above weight, then $D^{-}(\Gamma)>p$.

Theorem B. A sequence $\Gamma$ of distinct points in $\mathbb{D}$ is a set of (linear) interpolation for $v_{p}$ if and only if $\Gamma$ is uniformly discrete and $D^{+}(\Gamma)<p$.

Here $D^{+}(\Gamma)$ and $D^{-}(\Gamma)$ denote some special densities of $\Gamma$ independent of $p$ which will be defined below. Later, Berndtsson and Ortega Cerdà [BO, Thm. 6] extended the sufficiency part of Theorem B to weights $v$ for which $(-\Delta \log v(z)) \sim\left(1-|z|^{2}\right)^{-2}$. Their condition compares some functions of $z \in \mathbb{D}$ (and of an additional parameter $r$ ) depending on the sequence with functions depending on $-\Delta \log v$. Finally, Seip announced during ICM-98 in Berlin full characterizations for the same class of weights $v$. A rough sketch of the proof is contained in [S3, Thms. 2 and 3]. His approach is to embed $B_{v}^{\infty}$ into a suitable $B_{v_{p}}^{\infty}$ via a certain pointwise multiplier operator. The characterization is given in terms of more subtle new densities depending on $-\Delta \log v$.

Our paper has three main aims:

(1) To give a more elementary approach yielding an extension of Theorems A and B to more general not necessarily radial weights which allows us to evaluate the conditions of Seip from his characterization [S3]. We consider especially the family of weights $v: \Omega \rightarrow \mathbb{R}_{+}, \Omega$ an arbitrary simply connected domain, depending only on the distance from the boundary $\partial \Omega$ of $\Omega$, i.e., of the form $v(z)=\varphi(\operatorname{dist}(z, \partial \Omega))$. These are the most typical and interesting weights. Here we will express the conditions directly in terms of $\varphi$ (see Cor. 32, Thm. 10 and Cor. 11). A crucial role is played by Theorem 10.

(2) To get information on interpolating and sampling sequences for weights tending to zero at the boundary either very slowly or very rapidly (i.e., not contained in the class of $[\mathrm{BO}]$ ). We are interested in stability or a kind of uniform separation.

(3) To get new criteria of essentiality of non-radial weights, especially, weights depending on $\operatorname{dist}(z, \partial \Omega)$. A weight $v$ is essential if $v(z) \sim$ $1 /\left\|\delta_{z}\right\|$, where $\delta_{z}$ denotes the evaluation functional at $z$ and the norm is calculated in the dual of $B_{v}^{\infty}$. This notion plays a fundamental role in the theory of weighted Bergman spaces of infinite order (see $[\mathrm{BBT}],[\mathrm{BDL} 1],[\mathrm{AD}])$.

Last but not least, we prove some intrinsically interesting inequalities (which, we believe, will turn out useful in a more general context): see, for instance, Lemma 8 which leads to the crucial Theorem 10. 
In order to fulfill our first task we use a very simple comparison result (Proposition 21) which allows us to show that an interpolation (sampling) sequence for one weight has the same property for another weight. A novelty is that instead of using new densities depending on $v$ we measure decay of $v$ by some special indices. These indices give a rough estimate which sequences are interpolating or sampling for the given weight (Thm. 29). The indices are defined for arbitrary weights but they prove to be especially useful for the weights $v(z)=\varphi(\operatorname{dist}(z, \partial \Omega))$ (in particular, radial weights) because in that case they can be estimated by (or in some cases they are even equal to) indices of real positive functions $\varphi$ well-known from the theory of Orlicz spaces and convex functions ([Mal, p. 21], [LT, p. 143]) but so far not applied to Bergman spaces (see Theorem 10 and Corollary 11). Let us point out that the indices of weights are invariant with respect to biconformal maps, i.e., if $v: \Omega \rightarrow \mathbb{R}_{+}$is any weight and $f: \Omega_{0} \rightarrow \Omega$ a biconformal map, then the indices of $v$ and of $v \circ f$ coincide! Summarizing, although our results are not so general and precise as in [BO] or [S3], the conditions obtained are easier to check and cover also cases not included in the above-mentioned papers.

In that way we deduce, for instance:

(a) Theorems A and B hold for the whole class of weights which in some sense are similar to $v_{p}$ (Corollaries 31 and 32, cf. also Theorem 29 combined with Corollary 11). Note that this class contains a lot of spaces essentially different from $B_{v_{p}}^{\infty}$.

In order to fulfill the second task we apply our tools to get, for instance:

(b) If a weight $v$ tends to zero slowly, i.e., $U_{v}=0$ (so it is not contained in the class of $[\mathrm{BO}]$ ), then every interpolating sequence has $D^{+}$density zero (Corollary 30), is uniformly discrete (Corollary 16) and stable under perturbations (Lemma 18). The weights slowly tending to zero include logarithmic ones from [SW1, p. 265].

Moreover, we define a special metric $\varrho_{v}$ on $\Omega$ which allows us to get stability results for interpolating and sampling sequences also for arbitrary weights, even very rapidly tending to zero (see Corollary 16, Lemma 18). We obtain, for instance:

(c) If $v$ is an arbitrary weight, then every $H^{\infty}$ interpolating sequence is also a set of linear interpolation for $B_{v}^{\infty}$ (Corollary 23).

In the third area we prove three results: Theorem 4, Corollary 5 and Corollary 12. The best one is the last one:

(d) If $\varphi: \mathbb{R}_{+} \rightarrow \mathbb{R}_{+}$tends polynomially to zero at zero, then the weight $v(z):=\varphi(\operatorname{dist}(z, \partial \Omega))$ is essential. 
Our approach can be easily extended to several variables and is applicable to non-radial weights and general domains. Although our approach is different from Seip's [S3] we owe much to some ideas of [S2].

Observe that, in general, we cannot simplify our approach via simple tricks. The weights $v(z)=\varphi(\operatorname{dist}(z, \partial \Omega))$ can be transferred via the Riemann Mapping Theorem to the weights on $\mathbb{D}$ of the form

$$
w(z):=\varphi\left(\left(1-|z|^{2}\right)\left|f^{\prime}(z)\right|\right),
$$

where $f: \mathbb{D} \rightarrow \Omega$ is the Riemann map. As we will see later, if $\Omega$ is bounded and has the so-called Dini-smooth boundary, then $w \sim w_{1}$, where $w_{1}(z):=$ $\varphi\left(1-|z|^{2}\right)$, i.e., $w$ can be replaced by a radial weight. Unfortunately, this simple trick does not work for natural domains such as any half-plane or the strip $\Omega=\{z:|\operatorname{Im} z|<\pi / 2\}$. Moreover, if $\varphi(t)=t^{p}$, then the pointwise multiplication operator $M_{\left(f^{\prime}\right)^{p}}: B_{w}^{\infty} \rightarrow B_{w_{1}}^{\infty}$,

$$
M_{\left(f^{\prime}\right)^{p}}(g)(z):=\left(f^{\prime}(z)\right)^{p} g(z),
$$

is an onto isometry. This trick also allows one to get many results for $B_{w}^{\infty}$ but, unfortunately, it does not work for other functions $\varphi$. That is why we had to develop our theory.

The problem which sequences are sets of interpolation for various spaces of analytic functions has been studied extensively (see, for instance, [Sd, Sec. 11.5], [W, III.E], [BP], [M], [LS], [BO], [B], [GW], [S1], [Sch1], [Sch2], [ScS1], [ScS2]). The analogous problem for sampling sets has attracted much attention quite recently (see, for example, [LS], [GW], [S1], [Sch1], [Z2]).

The space $B_{v}^{\infty}$ appears naturally in the study of growth conditions for analytic functions and has been studied in many papers (see for example [RS], [SW1], [SW2], [BS], [L1], [L2], [BBT], [BDLT], [BDL1], [BDL2] and others). For references on Bergman spaces see [Z1] and especially [HKZ].

In Sections 3 and 4 we study in detail weights of moderate decay in the radial and non-radial case respectively. Such radial weights were considered by many authors using various defining conditions. Although it is probably known to specialists that some of them are equivalent we could not find any reference. That is why we collect them in Lemma 1 . We also collect known criteria for weights to be essential. We extend these results to weights on arbitrary $\Omega$ depending on the distance from the boundary. Then we introduce upper and lower indices $U_{v}$ and $L_{v}$ of an arbitrary weight $v$ and show how to calculate them easily for weights depending on the distance from the boundary.

In Section 5 we prove a useful result estimating the distance between the evaluation functionals on $B_{v}^{\infty}$ and we present results on stability of interpolating and sampling sequences in terms of a special metric $\varrho_{v}$ induced on $\mathbb{D}$ 
by the weight $v$. Section 6 contains comparison results and in Section 7 we obtain the main results characterizing interpolating and sampling sequences.

2. Preliminaries. A weight $v$ on the disc is radial if $v(z)=v(|z|)$. Every radial weight $v$ is assumed to be an almost decreasing function of $|z|$. As in $[\mathrm{T}]$ (cf. $[\mathrm{BBT}]$ ), a weight $v$ is called essential if there exists a $C>0$ such that

$$
v(z) \leq \widetilde{v}(z) \leq C v(z) \quad \text { for each } z,
$$

where the associated weight (see $[\mathrm{AD}]$ or $[\mathrm{BBT}]) \widetilde{v}$ is defined by

$$
\widetilde{v}(z):=\left(\sup \left\{|f(z)|: f \in B_{v}^{\infty},\|f\|_{v} \leq 1\right\}\right)^{-1} .
$$

If we take $\widetilde{v}$ instead of $v$, the space $B_{v}^{\infty}$ and the norm $\|\cdot\|_{v}$ do not change. The associated weight is better tied to the space $B_{v}^{\infty}$ than $v$ itself. For instance, for any sequence $\left(z_{n}\right) \subseteq \mathbb{D}$ and $f \in B_{v}^{\infty}$ the sequence $\left(f\left(z_{n}\right) \widetilde{v}\left(z_{n}\right)\right)$ is bounded. Roughly speaking, that means that one can expect sets of sampling or interpolation only for essential weights. Precisely, every set of (linear) interpolation or sampling for $v$ is a set of the same type for $\widetilde{v}$. Let us point out that $\widetilde{v}_{p}(z)=v_{p}(z), p>0$. Since every weight can be approximated by a smooth weight we may always pass to smooth weights without loss of generality. Note also that $-\log \widetilde{v}$ is subharmonic for every weight $v$.

We write $f \sim g$ for functions $f, g$ if there are positive constants $a, b$ such that $a f \leq g \leq b f$ for all values of variables. Functions $f, g$ are called additively equivalent, denoted by $f \mathbf{\uparrow} g$, if $|f-g|$ is bounded. We set $\Delta=\partial \bar{\partial}$, which differs from the standard definition of the Laplacian by a factor of 4 . The log-transform of a positive function $\varphi$ is $\Phi(t):=\log \varphi\left(e^{t}\right)$.

The pseudohyperbolic metric $\varrho$ on $\mathbb{D}$ is defined by

$$
\varrho(z, w):=\left|\varphi_{w}(z)\right|, \quad \text { where } \quad \varphi_{w}(z):=\frac{w-z}{1-\bar{w} z}, \quad z, w \in \mathbb{D} .
$$

A sequence $\left(z_{n}\right)$ in $\mathbb{D}$ is called d-uniformly discrete if $\inf _{n \neq k} d\left(z_{n}, z_{k}\right)>0$ for a given metric $d$. If $d=\varrho$ then we speak just about uniform discreteness. We denote by dist the Euclidean metric. A domain $\Omega$ is called thin if $\sup _{z \in \Omega} \operatorname{dist}(z, \partial \Omega)<\infty$. For instance, the strip $\{z:|\operatorname{Im} z|<\pi / 2\}$ is thin but unbounded.

For a uniformly discrete sequence $\Gamma=\left(z_{n}\right)$ and $1 / 2<r<1$, let

$$
D(\Gamma, r)=\left(\log \left(\frac{1}{1-r}\right)\right)^{-1} \sum_{1 / 2<\left|z_{n}\right|<r} \log \left(\frac{1}{\left|z_{n}\right|}\right) .
$$

The lower and upper uniform densities of $\Gamma$ are defined, respectively, as

$$
D^{-}(\Gamma)=\liminf _{r \rightarrow 1^{-}} \inf _{w \in \mathbb{D}} D\left(\varphi_{w}(\Gamma), r\right), \quad D^{+}(\Gamma)=\limsup _{r \rightarrow 1^{-}} \sup _{w \in \mathbb{D}} D\left(\varphi_{w}(\Gamma), r\right) .
$$


For an equivalent definition see [Sch2, Sec. 2]. These densities were introduced in [S2]. Closely related are the earlier notions proposed by Korenblum $[\mathrm{K}]$ in connection with his study of zero sets in $B_{v_{p}}^{\infty}$.

The classical interpolation problem in $H^{\infty}$ (i.e., for $v \equiv 1$ ) was completely solved by L. Carleson. It is known (see [W, III.E.4], [G, Ch. VIII]) that $\Gamma=\left(z_{n}\right)$ is a set of (linear) interpolation for $v(z)=1$ if and only if there is a $\delta>0$ such that for each fixed $k$,

$$
\prod_{n \neq k}\left|\frac{z_{n}-z_{k}}{1-\bar{z}_{n} z_{k}}\right| \geq \delta .
$$

If a set $\Gamma$ satisfies this condition, then $D^{+}(\Gamma)=0$ (see [Sch1, Lemma 5]).

3. Indices of radial weights of moderate decay. Recall that a function $f:[a, b] \rightarrow \mathbb{R}_{+}$is called almost decreasing (resp. increasing) if there exists a constant $C>0$ such that for any $x<y$ (resp. $x>y$ ) it follows that $f(y) \leq C f(x)$. It is worth noting that every almost decreasing (continuous) function on $[a, b]$ has an equivalent non-increasing majorant. There are three types of indices of a positive continuous function: at zero, at infinity and on $\mathbb{R}_{+}$. For our purposes we will use only the first and the last ones (see [Mal] $)$. We recall that the indices of positive continuous functions on $\left(0, t_{i}\right]$ (cf. [Mal, especially Thm. 3.3], [LT, p. 143]) are defined by

$$
\begin{aligned}
\alpha_{\varphi}^{i} & :=\sup \left\{q: \sup _{\lambda \in(0,1], t \in\left(0, t_{i}\right]} \frac{\varphi(\lambda t)}{\varphi(t) \lambda^{q}}<\infty\right\} \\
& =\sup \left\{q: \varphi(t) / t^{q} \text { is almost increasing for } t \in\left(0, t_{i}\right]\right\}, \\
\beta_{\varphi}^{i} & :=\inf \left\{q: \inf _{\lambda \in(0,1], t \in\left(0, t_{i}\right]} \frac{\varphi(\lambda t)}{\varphi(t) \lambda^{q}}>0\right\} \\
& =\inf \left\{q: t^{q} / \varphi(t) \text { is almost increasing for } t \in\left(0, t_{i}\right]\right\} .
\end{aligned}
$$

When $t_{i}=\infty$, we always consider $\left(0, t_{i}\right]=(0, \infty)$. If $t_{i}<\infty$ we may (and will) assume that $t_{i}=1$. In that case we denote the corresponding indices by $\alpha_{\varphi}^{0}, \beta_{\varphi}^{0}$ and call them the lower and upper index at zero. If $t_{i}=\infty$ we denote the indices by $\alpha_{\varphi}^{\infty}, \beta_{\varphi}^{\infty}$ and call them just the lower and upper index. We will use the notation $\alpha_{\varphi}^{i}, \beta_{\varphi}^{i}$ to cover both cases.

Clearly for $\varphi$ almost increasing we have $0 \leq \alpha_{\varphi}^{i} \leq \beta_{\varphi}^{i} \leq \infty$. We say that $\varphi$ satisfies the $\Delta_{2}$-condition (resp. the $\Delta_{2}$-condition at zero), briefly $\varphi \in \Delta_{2}^{\infty}$ ( $\varphi \in \Delta_{2}^{0}$, resp.), if there is a constant $C$ such that

$$
\varphi(2 t) \leq C \varphi(t) \quad \text { for } t \in \mathbb{R}_{+} \text {(for } t \in(0,1 / 2] \text {, resp). }
$$

For $\varphi$ almost increasing, it is easily seen that $\varphi \in \Delta_{2}^{\infty}$ (resp. $\varphi \in \Delta_{2}^{0}$ ) if and only if $\beta_{\varphi}^{\infty}<\infty\left(\beta_{\varphi}^{0}<\infty\right.$, resp. $)$.

Of course, every radial almost decreasing weight $v$ is of the form $v(z)=$ $\varphi(1-|z|)$, where $\varphi$ is almost increasing. We will denote the function $\varphi$ 
attached as above to $v$ by $\varphi_{v}$. Equivalently, $v(z)=\widehat{\varphi}\left(1-|z|^{2}\right)$, where $\widehat{\varphi}$ is also almost increasing. Since $\widehat{\varphi}(t)=\varphi(1-\sqrt{1-t})$ and $\varphi(2 t) \leq C \varphi(t)$ if and only if $\widehat{\varphi}(2 t) \leq \widehat{C} \widehat{\varphi}(t)$, we observe that $\beta_{\varphi}^{i}<\infty$ if and only if $\beta_{\widehat{\varphi}}^{i}<\infty$. Moreover, we easily observe that $\widehat{\varphi} \sim \varphi$ whenever the latter condition holds. Summarizing, if $\varphi \in \Delta_{2}$, then $\alpha_{\hat{\varphi}}^{i}=\alpha_{\varphi}^{i}$ and $\beta_{\hat{\varphi}}^{i}=\beta_{\varphi}^{i}$.

Some of the equivalent conditions in the next lemma are known but for the sake of completeness we give the whole proof. The conditions (a)(v) and (b)(iv) seem to be new.

LEMMA 1. Let $v$ be a radial weight on $\mathbb{D}, v(z)=\varphi(1-|z|)$, with $\varphi$ almost increasing, and set $\psi(t):=1 / v(1-1 / t), t \geq 1$.

(a) The following statements are equivalent:

(i) $\beta_{\varphi}^{0}<\infty$.

(ii) $\varphi$ satisfies the $\Delta_{2}$-condition at zero.

(iii) $v$ satisfies condition $(*)$ from $[\mathrm{L} 2$, p. 310], i.e.,

$$
\inf _{n} \frac{v\left(1-2^{-n-1}\right)}{v\left(1-2^{-n}\right)}>0 .
$$

(iv) $v$ satisfies condition $(U)$ from $[\mathrm{SW} 2$, p. 5], i.e., there exists a $q>0$ such that $\psi(x) / x^{q}$ is almost decreasing.

(v) $v$ has a bounded variation with respect to the pseudohyperbolic metric, i.e., there are $r>0$ and $C<\infty$ such that $v(z) / v(p) \leq C$ whenever $\varrho(z, p) \leq r$.

(b) The following statements are equivalent:

(i) $\alpha_{\varphi}^{0}>0$.

(ii) $v$ satisfies the condition from [L2, p. 310], i.e., there exists a $k \in \mathbb{N}$ such that

$$
\limsup _{n} \frac{v\left(1-2^{-n-k}\right)}{v\left(1-2^{-n}\right)}<1 .
$$

(iii) $v$ satisfies condition $(L)$ from $[\mathrm{SW} 2$, p. 5], i.e., there exists a $q>0$ such that $\psi(x) / x^{q}$ is almost increasing.

(iv) There is an $r \in(0,1)$ such that

$$
\lim _{|p| \rightarrow 1^{-}} \sup _{\varrho(z, p) \leq r} \frac{v(z)}{v(p)}>1 .
$$

In [SW2] Shields and Williams call weights $v$ satisfying both $(L)$ and $(U)$ (or, equivalently by Lemma $1,0<\alpha_{\varphi}^{0} \leq \beta_{\varphi}^{0}<\infty$ ) normal weights. Clearly the weight $v_{p}(z)=\left(1-|z|^{2}\right)^{p}, p>0$, is normal, $\alpha_{t^{p}}^{0}=\beta_{t^{p}}^{0}=p$. On the other hand, the weight $w(z)=(1-\log (1-|z|))^{-\varepsilon}, \varepsilon>0$, is not normal but satisfies condition $(U)$, in fact, $\beta_{\varphi_{w}}^{0}=0$. For $u(z)=\exp \left(A(1-|z|)^{B}\right)$ with $A, B<0$, we have $\alpha_{\varphi_{u}}^{0}=\beta_{\varphi_{u}}^{0}=\infty$. 
Proof of Lemma 1. We first show (a). It is easily seen that (i) $\Leftrightarrow($ ii), and (ii) $\Rightarrow$ (iii) follows by taking $t=2^{-n-1}$.

(iii) $\Rightarrow$ (i). By (iii), there is a $0<C<1$ with $v\left(1-2^{-n-1}\right) / v\left(1-2^{-n}\right)$ $>C$ for all $n$. Let $q:=-\log C / \log 2<\infty$. For given $\lambda, t \in(0,1]$, there exist $n, m \in \mathbb{N}$ with $2^{-m-1} \leq \lambda \leq 2^{-m}$ and $2^{-n-1} \leq t \leq 2^{-n}$. Thus $1-2^{-n} \leq 1-t \leq 1-2^{-n-1}$ and $1-2^{-m-n} \leq 1-\lambda t \leq 1-2^{-m-n-2}$. Now we have

$$
\frac{v(1-\lambda t)}{v(1-t) \lambda^{q}} \geq \frac{v\left(1-2^{-m-n-2}\right)}{v\left(1-2^{-n}\right) 2^{-m q}} \geq C^{m+2} 2^{m q}=C^{2} .
$$

Since $C$ does not depend on $\lambda$ and $t$ we conclude that $\beta_{\varphi}^{0}<\infty$.

(iv) $\Rightarrow$ (iii). Let $r=1-1 / t$, so $0 \leq r<1$. Then $v$ satisfies condition $(U)$ if and only if $(1-r)^{q} / v(r)$ is almost decreasing for some $q>0$. Putting $r=1-2^{-n}$ and $s=1-2^{-n-1}$ we obtain (iii).

(i) $\Rightarrow\left(\right.$ iv). By (i), there is a constant $C$ such that $v(1-\lambda t) /\left(v(1-t) \lambda^{q}\right)$ $\geq C$ for some $q<\infty$ and all $\lambda, t \in(0,1]$. For $0 \leq r<s<1$, we put $\lambda=(1-s) /(1-r)$ and $t=1-r$. Thus (iv) follows by using the same form of $(U)$ as above.

(iii) $\Leftrightarrow(v)$. First we observe that the pseudohyperbolic disc with center $p$ and radius $r$ is a Euclidean disc with diameter on the line through the origin and $p$, and intersects that line in the points

$$
\frac{|p|-r}{1-|p| r} \cdot \frac{p}{|p|} \quad \text { and } \quad \frac{|p|+r}{1+|p| r} \cdot \frac{p}{|p|}
$$

(cf. [Sh, Exercise 4.8.1]). If $z$ belongs to such a pseudohyperbolic disc and $r=$ $1 / 2$, then $1 / 3 \leq(1-|z|) /(1-|p|) \leq 3$. Thus, if $v\left(1-2^{-n-1}\right) / v\left(1-2^{-n}\right)>$ $C$ for all $n$, then for $r=1 / 2$ we have $v(z) / v(p)<C^{-2}$. On the other hand, if $v(p) / v(z) \leq C$ for $\varrho(z, p) \leq r$, then $v(1-t /(1+r)) / v(1-t) \geq 1 / C$ for all $t \in[0,1)$. Finally, for $k$ large enough,

$$
\frac{v\left(1-2^{-n-1}\right)}{v\left(1-2^{-n}\right)} \geq \frac{1}{C^{k}}
$$

(b) (i) $\Leftrightarrow$ (ii). If $\alpha_{\varphi}^{0}>0$, then there is a $C$ with $v(1-\lambda t) /\left(v(1-t) \lambda^{q}\right) \leq$ $C$ for some $q>0$ and all $\lambda, t \in(0,1]$. Fix $k$. Then $v\left(1-2^{-n-k}\right) / v\left(1-2^{-n}\right) \leq$ $C 2^{-k q}<\infty$ for all $n$. Thus $\limsup _{n} v\left(1-2^{-n-k}\right) / v\left(1-2^{-n}\right)<1$ for some $k \in \mathbb{N}$. Conversely, the assumption implies that there is an integer $k>0$, a constant $0<C<1$ and an index $n_{0} \geq 1$ with $v\left(1-2^{-n k-k}\right) / v\left(1-2^{-n k}\right) \leq$ $C$ for all $n \geq n_{0}$. Let $q:=-\log C /(k \log 2)>0$. It suffices to consider only $\lambda, t \in\left(0,2^{-\bar{n}_{0} k}\right]$. Given $\lambda, t \in\left(0,2^{-n_{0} k}\right]$, there exist $n, m \in \mathbb{N}$ with $n, m \geq n_{0}$ such that $2^{-(m+1) k} \leq \lambda \leq 2^{-m k}$ and $2^{-(n+1) k} \leq t \leq 2^{-n k}$. Consequently,

$$
\frac{v(1-\lambda t)}{v(1-t) \lambda^{q}} \leq \frac{v\left(1-2^{-(m+n) k}\right)}{v\left(1-2^{-(n+1) k}\right) 2^{-(m+1) k q}} \leq C^{m-1} 2^{(m+1) k q}=C^{-2}
$$


Again $C$ does not depend on $\lambda$ and $t$, so it follows that $\alpha_{\varphi}^{0}>0$.

The proof of (i) $\Rightarrow$ (iii) $\Rightarrow($ ii) $\Leftrightarrow$ (iv) is analogous to (a) (i) $\Rightarrow$ (iv) $\Rightarrow$ (iii) $\Leftrightarrow(\mathrm{v})$.

For later use we now collect known characterizations of essential not necessarily radial weights.

Proposition 2. Let $v$ be a strictly positive continuous function on $\mathbb{D}$.

(a) [BDL1, Prop. 7] If $v$ is a radial weight, then $v$ is essential if and only if it is equivalent to a log-convex radial function (weight) $w$, i.e., $t \mapsto$ $-\log w\left(e^{t}\right)$ is convex (or, for smooth functions, $-\Delta \log w(t)>0$ ).

(b) [SW2, Lemma 1] If $v$ is a radial continuous function and $\beta_{\varphi_{v}}^{0}<$ $\infty$, then $v$ is an essential weight if and only if it is almost decreasing (in particular, if $\left.\alpha_{\varphi_{v}}^{0}>0\right)$.

(c) [S3, Thm. 6] If $v$ is smooth and $-\Delta \log v(z) \sim\left(1-|z|^{2}\right)^{-2}$ near the boundary of $\mathbb{D}$, then $v$ is an essential weight.

Proof. (b) If $v$ is an essential weight, then by Observation 1.5 in [BBT] the associated weight $\widetilde{v}$ is decreasing and therefore $v$ is almost decreasing. If $\beta_{\varphi_{v}}^{0}<\infty$, the converse implication is a direct consequence of Lemma 1(iv) in [SW2] (cf. Prop. 3.4 in [BBT]). In [SW2] and [BBT], $v$ is assumed to be decreasing but the same proof works when $v$ is almost decreasing.

If $\alpha_{\varphi_{v}}^{0}>0$, then there are $q>0$ and $M$ such that $v(1-\lambda t) \leq M \lambda^{q} v(1-t)$ for all $\lambda, t \in(0,1]$. For $0 \leq r<s<1$, by putting $\lambda=(1-s) /(1-r)$ and $t=1-r$, we get $v(s) \leq M v(r)$.

(c) By [S3, Thm. 6] (cf. [S4]), we find two analytic functions $g_{1}, g_{2}$ such that $\left|g_{1}(z)\right| \sim \varrho\left(z, G_{1}\right) / v(z),\left|g_{2}(z)\right| \sim \varrho\left(z, G_{2}\right) / v(z)$, where $G_{1}, G_{2}$ are the zero sets of $g_{1}, g_{2}$, respectively, and $\varrho\left(G_{1}, G_{2}\right)>0$. It follows that $1 / v(z) \sim$ $\max \left(\left|g_{1}(z)\right|,\left|g_{2}(z)\right|\right)$.

TheOREM 3. For a radial weight $v$ on $\mathbb{D}$ we have $\beta_{\varphi_{v}}^{0}=\inf \{C>0: \exists w \sim v, w$ radial, smooth,

$$
\begin{aligned}
& \left.0 \leq \inf _{z \in \mathbb{D}}\left(1-|z|^{2}\right)^{2}(-\Delta \log w(z)) \leq \sup _{z \in \mathbb{D}}\left(1-|z|^{2}\right)^{2}(-\Delta \log w(z)) \leq C\right\} \\
= & \inf \left\{C>0: \exists w \sim v, w \text { smooth, } \sup _{z \in \mathbb{D}}\left(1-|z|^{2}\right)^{2}(-\Delta \log w(z)) \leq C\right\} .
\end{aligned}
$$

If $\beta_{\varphi_{v}}^{0}<\infty$, then

$\alpha_{\varphi_{v}}^{0}=\sup \{C \geq 0: \exists w \sim v, w$ radial, smooth,

$$
\begin{aligned}
& \left.C \leq \inf _{z \in \mathbb{D}}\left(1-|z|^{2}\right)^{2}(-\Delta \log w(z)) \leq \sup _{z \in \mathbb{D}}\left(1-|z|^{2}\right)^{2}(-\Delta \log w(z))<\infty\right\} \\
= & \sup \left\{C \geq 0: \exists w \sim v, w \text { smooth, } C \leq \inf _{z \in \mathbb{D}}\left(1-|z|^{2}\right)^{2}(-\Delta \log w(z))\right\} .
\end{aligned}
$$


We omit the proof since later we prove a more general result (see Theorem 10 and Corollary 11).

4. General weights of moderate decay. Apart from radial weights on the unit disc another class of natural weights are those weights on an arbitrary simply connected domain $\Omega$ which depend on the distance from the boundary. We will try to develop their theory in the spirit of the theory of radial weights. We always denote by $g: \Omega \rightarrow \mathbb{D}$ a Riemann map and by $f$ its inverse. By $\operatorname{dist}(z, \partial \Omega)$ we denote the Euclidean distance of $z$ to the boundary of $\Omega$. By [Po, Cor. 1.4],

$$
\begin{aligned}
\frac{1-|g(z)|^{2}}{4\left|g^{\prime}(z)\right|} & \leq \operatorname{dist}(z, \partial \Omega) \leq \frac{1-|g(z)|^{2}}{\left|g^{\prime}(z)\right|} \\
\frac{1}{4}\left(1-|z|^{2}\right)\left|f^{\prime}(z)\right| & \leq \operatorname{dist}(f(z), \partial \Omega) \leq\left(1-|z|^{2}\right)\left|f^{\prime}(z)\right| .
\end{aligned}
$$

Note that the rightmost term in the first line above is closely connected to the Bergman kernel of $\Omega$. Thus for $\varphi$ almost increasing and $\varphi \in \Delta_{2}^{\infty}$ the weights

$$
\varphi(\operatorname{dist}(z, \partial \Omega)) \text { and } \varphi\left(\frac{1-|g(z)|^{2}}{\left|g^{\prime}(z)\right|}\right)
$$

are equivalent; similarly, after transferring the weights via the Riemann mapping $f$ to the disc, the weights

$$
\varphi(\operatorname{dist}(f(z), \partial \Omega)) \quad \text { and } \quad \varphi\left(\left(1-|z|^{2}\right)\left|f^{\prime}(z)\right|\right)
$$

are equivalent. If the domain $\Omega$ is thin (i.e., $\sup _{z \in \Omega} \operatorname{dist}(z, \partial \Omega)<\infty$ ), then it suffices to assume that $\varphi \in \Delta_{2}^{0}$. If the boundary of $\Omega$ is a Dini-smooth Jordan curve, then $\left|f^{\prime}\right|$ is bounded from above and from below [Po, Thm. 3.5]. Thus in that case the weights above are equivalent to radial weights. Let us point out that this does not apply to any unbounded domain (like a half-plane or strip).

Since the general form of the biholomorphic map $\varphi: \mathbb{D} \rightarrow \mathbb{D}$ is given by $\varphi(z)=e^{i \theta}(w-z) /(1-\bar{w} z)$ and

$$
\frac{|\partial \varphi / \partial z|^{2}}{\left(1-|\varphi(z)|^{2}\right)^{2}}=\frac{1}{\left(1-|z|^{2}\right)^{2}}
$$

the following definition does not depend on the choice of the Riemann map $g: \Omega \rightarrow \mathbb{D}$. Let $v$ be a continuous weight on a simply connected domain $\Omega \subseteq \mathbb{C}, \Omega \neq \mathbb{C}$. Then we define the upper and lower index of the weight $v$ by

$$
\begin{array}{r}
U_{v}:=\inf \left\{C>0: \exists w \sim v, w \text { smooth, }-\infty<\inf _{z \in \Omega}(-\Delta \log w(z)) \frac{\left(1-|g(z)|^{2}\right)^{2}}{|\partial g / \partial z|^{2}}\right. \\
\left.\leq \sup _{z \in \Omega}(-\Delta \log w(z)) \frac{\left(1-|g(z)|^{2}\right)^{2}}{|\partial g / \partial z|^{2}} \leq C\right\},
\end{array}
$$




$$
\begin{array}{r}
L_{v}:=\sup \left\{C \geq 0: \exists w \sim v, w \text { smooth, } \infty>\sup _{z \in \Omega}(-\Delta \log w(z)) \frac{\left(1-|g(z)|^{2}\right)^{2}}{|\partial g / \partial z|^{2}}\right. \\
\left.\geq \inf _{z \in \Omega}(-\Delta \log w(z)) \frac{\left(1-|g(z)|^{2}\right)^{2}}{|\partial g / \partial z|^{2}} \geq C\right\} .
\end{array}
$$

By Theorem 3, the above definition coincides for radial weights on $\mathbb{D}$ with the previous definition. That means that if $v$ is radial and $\beta_{\varphi_{v}}^{0}<\infty$, then $v$ is automatically essential by Proposition 2(b) and therefore

$$
U_{v}=\beta_{\varphi_{v}}^{0} \quad \text { and } \quad L_{v}=\alpha_{\varphi_{v}}^{0} .
$$

Since $\Delta(f \circ h)=(\Delta f) \cdot|\partial h / \partial z|^{2}$ for $h$ analytic, the above definition is invariant with respect to transferring weights via the Riemann map, i.e., if $f: \Omega_{1} \rightarrow \Omega_{2}$ is a biholomorphic map and $v: \Omega_{2} \rightarrow \mathbb{R}_{+}$is a weight, then

$$
U_{v}=U_{v \circ f}, \quad L_{v}=L_{v \circ f} .
$$

Note that if $\Omega$ is the upper half-plane, then

$$
\frac{\left(1-|g(z)|^{2}\right)^{2}}{|\partial g / \partial z|^{2}}=4|\operatorname{Im} z|^{2}
$$

Similarly, if $\Omega=\{z \in \mathbb{C}:|\operatorname{Im} z|<\pi / 2\}$, then

$$
\frac{\left(1-|g(z)|^{2}\right)^{2}}{|\partial g / \partial z|^{2}}=4 \cos ^{2}(\operatorname{Im} z) \text {. }
$$

Moreover, if $h$ is non-vanishing and analytic, then $-\Delta \log \left(\left(1-|z|^{2}\right)|h(z)|\right)^{\alpha}=$ $\alpha /\left(1-|z|^{2}\right)^{2}$, which allows one to calculate the indices $U_{v}$ and $L_{v}$ easily for that weight on the disc. This suggests that we can calculate the indices easily for weights depending on $\left(1-|z|^{2}\right)|h(z)|$.

The following result is a generalization of Proposition 2(b) above. It is especially interesting for $h=f^{\prime}$ (see above).

TheOREM 4. Let $h: \mathbb{D} \rightarrow \mathbb{C}$ be an analytic function without zeros such that a branch of the argument of $h$ is bounded. Let $\varphi: \mathbb{R}_{+} \rightarrow \mathbb{R}_{+}$be an almost increasing continuous function with the $\Delta_{2}^{\infty}$-condition. If the weight $v(z):=\varphi\left(\left(1-|z|^{2}\right)|h(z)|\right)$ is bounded, then it is essential. If $\left(1-|z|^{2}\right)|h(z)|$ is bounded then it suffices to assume the $\Delta_{2}$-condition only at zero.

The problem appears for which Riemann maps $f$ their derivative $f^{\prime}$ has a bounded branch of argument. By [Po, Thm. 3.2], if the boundary of $\Omega$ is a smooth Jordan curve (i.e., in particular, $\Omega$ is bounded), then the argument of $f^{\prime}$ is bounded (since it extends continuously to the closed disc). For general (bounded) $\Omega$, it is not true. For instance, take

$$
\Omega:=\mathbb{D} \backslash(\{\gamma(t): t \geq 1\} \cup\{0\}),
$$

where $\gamma(t)=t^{-1} e^{i t}$. In general, the argument of $f^{\prime}$ at $r e^{i \theta}$ can be calculated as $\pi / 2+u+\theta$, where $u$ is an argument of the derivative of the curve $\theta \mapsto$ 
$f\left(r e^{i \theta}\right)$. So if the arguments of the derivative of all these curves are bounded then the assumption of Theorem 4 is satisfied for $h=f^{\prime}$. This gives plenty of examples of simply connected (bounded or unbounded) domains $\Omega$ with bounded argument of $f^{\prime}$ (even without any smoothness of the boundary). For instance, by [Po, p. 66, formula (5)], if $\Omega$ is a star-like domain with respect to zero (or its translation, for instance any convex domain), then $f(r \mathbb{D})$ are also star-like and then one observes easily that $f^{\prime}$ has a bounded branch of argument.

Corollary 5. Let $\Omega$ be a thin domain such that the argument of the derivative $f^{\prime}$ of the Riemann map $f: \mathbb{D} \rightarrow \Omega$ is bounded. If an almost increasing continuous function $\varphi: \mathbb{R}_{+} \rightarrow \mathbb{R}_{+}$satisfies the $\Delta_{2}$-condition at zero, then the weight

$$
v(z):=\varphi(\operatorname{dist}(z, \partial \Omega))
$$

on $\Omega$ is essential.

Proof of Theorem 4. We may assume that $v$ is bounded by 1 . Define

$$
k(z):=e^{i \theta}\left(h(z)\left(1-e^{i \lambda} z^{2}\right)\right)^{-\alpha},
$$

where $\alpha>0$ is chosen in such a way that for every $\lambda$ there is $\theta$ such that $k: \mathbb{D} \rightarrow H_{+}:=\{z: \operatorname{Re} z>0\}$. Moreover, for $t \in[1 / 2,1), p>0, C>0$, we define an analytic function $s_{t}: \mathbb{D} \rightarrow \mathbb{C}$ by

$$
s_{t}(z):=C\left(\frac{1}{1-t\left(1-k(z)^{-1}\right)}\right)^{p} .
$$

Fix $z_{0} \in \mathbb{D}$ and define $t_{0}<1$ by

$$
\frac{1}{1-t_{0}}=\left|h\left(z_{0}\right)\left(1-\left|z_{0}\right|^{2}\right)\right|^{-\alpha} .
$$

We will show the following properties of $s_{t}$ whenever $t_{0} \geq 1 / 2$ :

$$
\begin{gathered}
\left|s_{t}(z)\right| \leq 2^{p} C\left|h(z)\left(1-|z|^{2}\right)\right|^{-p \alpha} \quad \text { for } t \geq 1 / 2 \text { and } z \in \mathbb{D}, \\
\left|s_{t_{0}}(z)\right| \leq C\left|h\left(z_{0}\right)\left(1-\left|z_{0}\right|^{2}\right)\right|^{-p \alpha} \quad \text { for } z \in \mathbb{D}, \\
\left|s_{t_{0}}\left(z_{0}\right)\right| \geq \frac{C}{2^{p}}\left|k\left(z_{0}\right)\right|^{p}
\end{gathered}
$$

We start with the proof of (2). It is easily seen that for $\operatorname{Re} w \geq 0$ and $t \geq 1 / 2$ we have $|(1-t)+t w| \geq \frac{1}{2}|w|$. Thus

$$
\frac{1}{|k(z)|} \leq 2\left|(1-t)+\frac{t}{k(z)}\right| .
$$

Since $1-|z|^{2} \leq\left|1-e^{i \lambda} z^{2}\right|$ we have

$$
|k(z)| \leq\left(|h(z)|\left(1-|z|^{2}\right)\right)^{-\alpha} .
$$

Combining the above inequalities we obtain (2). 
In order to show (3) it suffices to observe that

$$
\left|1-t_{0}+\frac{t_{0}}{k(z)}\right|^{-1} \leq\left(\operatorname{Re}\left(\left(1-t_{0}\right)+\frac{t_{0}}{k(z)}\right)\right)^{-1} \leq \frac{1}{1-t_{0}} .
$$

To obtain (4) we note that

$$
\frac{C\left|k\left(z_{0}\right)\right|^{p}}{\left|s_{t_{0}}\left(z_{0}\right)\right|}=\left|\left(1-t_{0}\right) k\left(z_{0}\right)+t_{0}\right|^{p} \leq\left(1+t_{0}\right)^{p} \leq 2^{p} .
$$

Now, we are ready to prove the result. Modifying suitably $\varphi$ we may find $q>0$ such that $t^{q} / \varphi(t)$ is a strictly increasing function. Let $p$ satisfy $p \alpha=q$. Then we take an arbitrary $z_{0} \in \mathbb{D}$ and find $t_{0}$ according to (1). For $\lambda$ satisfying $e^{i \lambda} z_{0}^{2} \geq 0$ we take $\theta$ in such a way that $k(\mathbb{D}) \subseteq H_{+}$. Since $\varphi$ is almost increasing we assume that

$$
\varphi\left(t_{1}\right) \leq C_{1} \varphi\left(t_{2}\right) \quad \text { for } t_{1}<t_{2} .
$$

If $t_{0} \leq 1 / 2$, then $v\left(z_{0}\right) \geq C_{1}^{-1} \varphi\left(2^{-1 / \alpha}\right)=: C_{2}$, where the latter constant does not depend on $z_{0}$. The function $f \equiv 1$ satisfies $\|f\|_{v}=1=\sup _{z \in \mathbb{D}} v(z)$ and $\left|f\left(z_{0}\right) v\left(z_{0}\right)\right| \geq C_{2}$.

If $t_{0} \geq 1 / 2$, then we take $C>0$ such that

$$
\varphi\left(\left|h\left(z_{0}\right)\right|\left(1-\left|z_{0}\right|^{2}\right)\right)=C^{-1}\left(\left|h\left(z_{0}\right)\right|\left(1-\left|z_{0}\right|^{2}\right)\right)^{q} .
$$

Then we define $f:=s_{t_{0}}$. By (4) and the choice of the constants $\lambda$ and $C$, we have

$$
\left|f\left(z_{0}\right)\right| \geq 2^{-p} v\left(z_{0}\right)^{-1}
$$

If $\left|h(z)\left(1-|z|^{2}\right)\right| \geq\left|h\left(z_{0}\right)\left(1-\left|z_{0}\right|^{2}\right)\right|$, then $v(z)^{-1} \geq C\left|h(z)\left(1-|z|^{2}\right)\right|^{-q}$ (because $t^{q} / \varphi(t)$ is increasing). Therefore, by (2),

$$
|f(z)| \leq 2^{p} v(z)^{-1} \text {. }
$$

If $\left|h(z)\left(1-|z|^{2}\right)\right| \leq\left|h\left(z_{0}\right)\left(1-\left|z_{0}\right|^{2}\right)\right|$, then $C_{1} v(z)^{-1} \geq v\left(z_{0}\right)^{-1}$ (because $\varphi$ is almost increasing). By (3), $|f(z)| \leq C_{1} v(z)^{-1}$.

Summarizing, $\|f\|_{v} \leq \max \left(2^{p}, C_{1}, 1\right)$ but $\left|f\left(z_{0}\right)\right| v\left(z_{0}\right) \geq \min \left(2^{-p}, C_{2}\right)$. The weight is essential.

Now, we prove an analogue of Theorem 3 showing that for weights depending only on the distance to the boundary the indices are easily calculable.

First we need some definitions. Recall that the log-transform of the function $\varphi: \mathbb{R}_{+} \rightarrow \mathbb{R}_{+}$is the function $\Phi: \mathbb{R} \rightarrow \mathbb{R}$ given by

$$
\Phi(t):=\log \varphi\left(e^{t}\right) .
$$

Further, recall that the functions $f, g$ are called additively equivalent, denoted by $f \boldsymbol{\uparrow} g$, if $|f-g|$ is bounded.

Lemma 6. If $F, G: A \rightarrow \mathbb{R}, A=\mathbb{R}$ or $A=a$ half-line, are additively equivalent continuously differentiable functions such that $F^{\prime} \leq a, G^{\prime} \geq b$ 
then there is a continuously differentiable function $H: A \rightarrow \mathbb{R}$ additively equivalent to $F$ and $G$ such that

$$
b \leq H^{\prime} \leq a .
$$

Proof. We give a proof only for $A=\mathbb{R}$. If $G^{\prime} \geq F^{\prime}$ then we take

$$
H(t)=\int_{0}^{t}\left(\min \left(G^{\prime}(x), a\right)+G(0)\right) d x \quad \text { for } t \in \mathbb{R} .
$$

This is the function we are looking for since $F^{\prime} \leq H^{\prime} \leq G^{\prime}$. If $G^{\prime} \leq a$, then we take $H=G$.

Let $I:=\left\{t: G^{\prime}(t)>a\right\}$. This is a union of countably many open intervals. Since $G^{\prime}$ is uniformly continuous on compact sets, there are only finitely many of these intervals intersecting $[-n,-n+1] \cup[n-1, n], n \in \mathbb{N}$, and such that $G^{\prime}>a+2^{-n}$ somewhere in the interval. Therefore we may modify $G^{\prime}$ in such a way that $G \mathbf{\$} F, G^{\prime} \geq b$ but

$$
\left\{t \in[-n, n]: G^{\prime}(t)>a\right\}
$$

is a union of finitely many open intervals. Indeed, it suffices to cut off "small bumps" of $G^{\prime}$ over $a$ and integrate.

Assume that $I \cap \mathbb{R}_{+}=\bigcup_{n \in \mathbb{N}}\left(p_{n}, k_{n}\right)$, with the intervals $\left(p_{n}, k_{n}\right)$ numbered in order of increasing starting points. Without loss of generality we assume that $p_{0}=0$. We define $h(t)=a$ for $t \in\left(p_{n}, k_{n}\right), n \in \mathbb{N}$. Let

$$
t_{1}:=\inf \left\{t: \int_{0}^{t}\left(F^{\prime}-G^{\prime}\right)^{+}=\int_{0}^{t}\left(G^{\prime}-a\right)^{+}\right\} .
$$

Then for $t \in\left(0, t_{1}\right)$, we define

$$
h(t):=\max \left(F^{\prime}(t), \min \left(G^{\prime}(t), a\right)\right) .
$$

Of course, $t_{1} \in\left(k_{n}, p_{n+1}\right)$ and for $t \in\left(t_{1}, p_{n+1}\right)$ we define

$$
h(t):=G^{\prime}(t) .
$$

Then we repeat the same procedure starting from $p_{n+1}$ instead of 0 . Inductively, we define $h(t)$ for $t>0$. An analogous procedure gives $h(t)$ for negative $t$.

We define

$$
H(t):=G(0)+\int_{0}^{t} h(x) d x \quad \text { for } t \in \mathbb{R} .
$$

Clearly, $b \leq H^{\prime} \leq a$ and $H$ is differentiable. For $t \in\left(0, t_{1}\right), F^{\prime}(t) \leq H^{\prime}(t)$. Thus

$$
(G(0)-F(0))+F(t) \leq H(t) .
$$


On the other hand, $H(t) \leq G(t)$ because for $u<t_{1}$,

$$
\begin{aligned}
\int_{0}^{u}\left(h-G^{\prime}\right) & =\int_{[0, u] \backslash I}\left(F^{\prime}(t)-G^{\prime}(t)\right)^{+} d t-\int_{[0, u] \cap I}\left(G^{\prime}(t)-a\right)^{+} d t \\
& =\int_{0}^{u}\left(F^{\prime}-G^{\prime}\right)^{+}-\int_{0}^{u}\left(G^{\prime}-a\right)^{+} \leq 0 .
\end{aligned}
$$

Since $\int_{0}^{t_{1}}\left(h-G^{\prime}\right)=0$, we have $H\left(t_{1}\right)=G\left(t_{1}\right)$ and the same holds for other $t \in\left(t_{1}, p_{n+1}\right)$. Repeating the above procedure inductively we get

$$
F(t)-\sup _{u}|F(u)-G(u)| \leq H(t) \leq G(t) .
$$

Lemma 7. If $H: A \rightarrow \mathbb{R}, A=\mathbb{R}$ or $A=a$ half-line, is a continuously differentiable function such that

$$
b \leq H^{\prime} \leq a
$$

then for every $\varepsilon>0$ there is a smooth function $G: A \rightarrow \mathbb{R}$ additively equivalent to $H$ such that

$$
\left|G^{\prime \prime}\right|<\varepsilon, \quad b \leq G^{\prime} \leq a .
$$

Proof. We prove the lemma only for $A=\mathbb{R}_{+}$. We take $k=(a-b) / \varepsilon$ and define a piecewise affine continuous function $h$ with knots at $l k, l \in \mathbb{N}$, as follows. We take $h(0)=b$ and set

$$
r(l)=\frac{2}{k} \int_{0}^{l k}\left(H^{\prime}-b\right)-2\left(\sum_{j=1}^{l-1}(h(j k)-b)\right)+b
$$

and

$$
h(l k):= \begin{cases}a & \text { if } r(l)>a, \\ r(l) & \text { if } r(l) \in[b, a], \\ b & \text { if } r(l)<b .\end{cases}
$$

Between multiples of $k$ we define $h$ to be affine. Note that if $r(l) \in[b, a]$, then

$$
\int_{0}^{l k}\left(h(u)-H^{\prime}(u)\right) d u=0
$$

and for $t \in[l k,(l+1) k]$ we have

$$
\left|\int_{0}^{t}\left(h(u)-H^{\prime}(u)\right) d u\right|=\left|\int_{l k}^{t}\left(h(u)-H^{\prime}(u)\right) d u\right| \leq \frac{(a-b) k}{2} .
$$

If $r(l+1), \ldots, r(l+m)>a$, then $h(t)=a$ for $t \in[(l+1) k,(l+m) k]$ so 
$h(t) \geq H^{\prime}(t)$ for these $t$ but still

$$
\int_{l k}^{t}\left(h(u)-H^{\prime}(u)\right) d u \leq 0 .
$$

Therefore $\int_{l k}^{t}\left(h(u)-H^{\prime}(u)\right) d u$ is a non-decreasing function of $t$ and it is not less than $-(a-b) k / 2$. Treating the case of $r(l)<b$ analogously, we can show that

$$
\left|\int_{0}^{t}\left(h(u)-H^{\prime}(u)\right) d u\right| \leq 2(a-b) k .
$$

Since $\left|h^{\prime}\right| \leq \varepsilon$ whenever $h^{\prime}$ exists, we can find a smooth function $\widetilde{h}$ such that $b \leq \widetilde{h} \leq a,\left|\widetilde{h}^{\prime}\right| \leq \varepsilon$ and $\int_{\mathbb{R}}|h-\widetilde{h}|<1$. Thus

$$
G(t):=\int_{0}^{t} \widetilde{h}(u) d u+H(0)
$$

is a function we are looking for.

Now, we prove a crucial lemma.

Lemma 8. Let $\varphi: \mathbb{R}_{+} \rightarrow \mathbb{R}_{+}$be almost increasing and let $\Phi$ denote the log-transform of $\varphi$. If $f: \mathbb{D} \rightarrow \Omega$ is a conformal equivalence, then the weight

$$
v(z):=\varphi\left(\left(1-|z|^{2}\right)\left|f^{\prime}(z)\right|\right)
$$

satisfies the following inequality:

$$
\left|-\Delta \log v(z)-\frac{\Phi^{\prime}(k(z))}{\left(1-|z|^{2}\right)^{2}}\right| \leq \frac{4\left|\Phi^{\prime \prime}(k(z))\right|}{\left(1-|z|^{2}\right)^{2}},
$$

where

$$
k(z):=\log \left(\left(1-|z|^{2}\right)\left|f^{\prime}(z)\right|\right) .
$$

Proof. We have

$$
-\Delta \log v(z)=-\Delta \log \varphi(\exp (k(z)))=-\Delta \Phi \circ k(z) .
$$

Moreover,

$$
\Delta \Phi \circ k(z)=\Phi^{\prime \prime}(k(z)) \cdot(\partial k(z) \bar{\partial} k(z))+\Phi^{\prime}(k(z)) \cdot \Delta k(z) .
$$

We calculate

$$
\begin{aligned}
& \partial k(z)=\frac{-\bar{z}}{1-|z|^{2}}+\frac{1}{2} \frac{f^{\prime \prime}(z) \cdot \overline{f^{\prime}(z)}}{\left|f^{\prime}(z)\right|^{2}}, \\
& \bar{\partial} k(z)=\frac{-z}{1-|z|^{2}}+\frac{1}{2} \frac{\overline{f^{\prime \prime}(z)} \cdot f^{\prime}(z)}{\left|f^{\prime}(z)\right|^{2}} .
\end{aligned}
$$

Since $f^{\prime}$ never vanishes we have

$$
\Delta k(z)=\Delta \log \left(1-|z|^{2}\right)=\frac{-1}{\left(1-|z|^{2}\right)^{2}} .
$$


Therefore, we obtain

$$
\begin{aligned}
-\Delta \log v(z) & =-\Delta \Phi \circ k(z) \\
& =-\Phi^{\prime \prime}(k(z)) \cdot\left|\frac{-\bar{z}}{1-|z|^{2}}+\frac{1}{2} \frac{f^{\prime \prime}(z)}{f^{\prime}(z)}\right|^{2}+\Phi^{\prime}(k(z)) \frac{1}{\left(1-|z|^{2}\right)^{2}} \\
& =\frac{1}{\left(1-|z|^{2}\right)^{2}}\left(\Phi^{\prime}(k(z))-\Phi^{\prime \prime}(k(z)) \cdot\left|\frac{1}{2} \frac{f^{\prime \prime}(z)}{f^{\prime}(z)}\left(1-|z|^{2}\right)-\bar{z}\right|^{2}\right) .
\end{aligned}
$$

By [Po, Prop. 1.2] (which follows immediately from the Bieberbach conjecture for the second coefficient),

$$
\left|\frac{1}{2} \frac{f^{\prime \prime}(z)}{f^{\prime}(z)}\left(1-|z|^{2}\right)-\bar{z}\right| \leq 2 \quad \text { for } z \in \mathbb{D} .
$$

Thus the required inequality follows.

Lemma 9. Let $\varphi: \mathbb{R}_{+} \rightarrow \mathbb{R}_{+}$be a differentiable function and let $\Phi$ be its log-transform. Let $t_{0}=1, t_{\infty}=\infty$. Then, for $i=0$ or $i=\infty$,

(a) $\alpha_{\varphi}^{i}=\sup \left\{\inf _{t \in\left(0, t_{i}\right]} \Phi_{1}^{\prime}(t): \Phi \boldsymbol{\phi} \Phi_{1}\right.$ and $\Phi_{1}$ is smooth $\}$;

(b) $\beta_{\varphi}^{i}=\inf \left\{\sup _{t \in\left(0, t_{i}\right]} \Phi_{1}^{\prime}(t): \Phi \boldsymbol{\Lambda} \Phi_{1}\right.$ and $\Phi_{1}$ is smooth $\}$;

(c) $\varphi$ is almost increasing (near zero) if and only if there exists $\Phi_{1} \boldsymbol{\Lambda} \Phi$, $\Phi_{1}$ smooth, such that $\Phi_{1}^{\prime} \geq 0$ (near zero).

Proof. (a) By the definition, it follows easily that

$\alpha_{\varphi}^{i}=\sup \left\{q: \exists \varphi_{1} \sim \varphi: \varphi_{1}(t) / t^{q}\right.$ is increasing, $t \in\left(0, t_{i}\right], \varphi_{1}$ smooth $\}$.

Thus for the $\log$-transform $\Phi_{1}$ of $\varphi_{1}$ we see that

$$
\log \left(\frac{\varphi_{1}\left(e^{t}\right)}{e^{t q}}\right)=\Phi_{1}(t)-t q
$$

is increasing, hence $\Phi_{1}^{\prime}(t) \geq q$.

The proof of (b) and (c) is analogous.

TheOREM 10. Let $f: \mathbb{D} \rightarrow \Omega$ be a biconformal map. Let $\varphi: \mathbb{R}_{+} \rightarrow \mathbb{R}_{+}$ be an almost increasing function with $\beta_{\varphi}^{i}<\infty$, where $i=0$ or $\infty$ depending on whether $\left(1-|z|^{2}\right)\left|f^{\prime}(z)\right|$ is bounded on $\mathbb{D}$ or not (i.e., whether $\Omega$ is thin or not). Then for every $\varepsilon>0$ there is a smooth weight $w$ depending on $\left(1-|z|^{2}\right)\left|f^{\prime}(z)\right|$ and equivalent to $v(z):=\varphi\left(\left(1-|z|^{2}\right)\left|f^{\prime}(z)\right|\right)$ such that

$$
\frac{\alpha_{\varphi}^{i}-\varepsilon}{\left(1-|z|^{2}\right)^{2}} \leq-\Delta \log w(z) \leq \frac{\beta_{\varphi}^{i}+\varepsilon}{\left(1-|z|^{2}\right)^{2}}
$$

Proof. By Lemma 9, there are two functions $\Phi_{1}, \Phi_{2}$ additively equivalent to the $\log$-transform of $\varphi$ such that

$$
\Phi_{1}^{\prime} \geq \alpha_{\varphi}^{i}-\varepsilon / 2 \quad \text { and } \quad \Phi_{2}^{\prime} \leq \beta_{\varphi}^{i}+\varepsilon / 2 .
$$


Applying Lemmas 6 and 7 we find another smooth function $\Psi$ additively equivalent to the log-transform of $\varphi$ such that

$$
\alpha_{\varphi}^{i}-\varepsilon / 2 \leq \Psi^{\prime} \leq \beta_{\varphi}^{i}+\varepsilon / 2 \text { and }\left|\Psi^{\prime \prime}\right| \leq \varepsilon / 8 .
$$

By Lemma 8, the weight

$$
w(z):=\exp \left(\Psi\left(\log \left(\left(1-|z|^{2}\right)\left|f^{\prime}(z)\right|\right)\right)\right)
$$

is the one we are looking for.

Corollary 11. If $\varphi$ is an almost increasing function, $\varphi \in \Delta_{2}^{\infty}$, and $v$ is a weight on some simply connected domain $\Omega \subseteq \mathbb{C}, v(z)=\varphi(\operatorname{dist}(z, \partial \Omega))$, then

$$
\alpha_{\varphi}^{\infty} \leq L_{v} \leq U_{v} \leq \beta_{\varphi}^{\infty} .
$$

If $\Omega$ is bounded then $U_{v}=\beta_{\varphi}^{0}$ and if , additionally, $\varphi \in \Delta_{2}^{0}$ then $L_{v}=\alpha_{\varphi}^{0}$.

Proof. If $\Omega$ is bounded, then the curves $\left(1-|g(z)|^{2}\right) /\left|g^{\prime}(z)\right|=$ const are closed. Let

$$
-\Delta \log w(z) \leq C\left(1-|z|^{2}\right)^{-2} \quad \text { for } w \sim v, v(z)=\varphi(H(z)),
$$

and

$$
H(z):=\frac{1-|g(z)|^{2}}{|\partial g / \partial z|} .
$$

Thus $\log \left(w(z) / H(z)^{C}\right)$ is subharmonic, so $w(z) / H(z)^{C}$ is either constant or has no local maximum. If $t^{C} / \varphi(t)$ is not almost increasing then we can find sequences $\left(t_{1, n}\right),\left(t_{2, n}\right), t_{1, n}<t_{2, n}$, in the range of $\varphi$ such that $t_{1, n}^{C} / \varphi\left(t_{1, n}\right)>n t_{2, n}^{C} / \varphi\left(t_{2, n}\right)$. This leads to a contradiction by taking $n$ large enough with respect to the equivalence constant of $w$ and $v$ because $\Omega$ is thin and therefore $H$ is bounded. A similar proof works for $L_{v}=\alpha_{\varphi}^{0}$.

Corollary 12. If $\Omega$ is a simply connected domain, $\varphi$ is almost increasing and $0<\alpha_{\varphi}^{\infty} \leq \beta_{\varphi}^{\infty}<\infty$, then the weight

$$
v(z)=\varphi(\operatorname{dist}(z, \partial \Omega))
$$

is essential. For thin domains it suffices to assume $0<\alpha_{\varphi}^{0} \leq \beta_{\varphi}^{0}<\infty$.

Proof. By Theorem 10, there is a smooth weight $w$ equivalent to $v$ such that

$$
-\Delta \log w(z) \sim \frac{1}{\left(1-|z|^{2}\right)^{2}} .
$$

By Proposition 2(c), the result follows.

Corollary 11 cannot be improved. Take $\varphi(t):=e^{t} t$ and consider the weight $w(z):=\varphi(|\operatorname{Re} z|)$ on the left half-plane $\operatorname{Re} z<0$. Via the Riemann map we obtain a weight on $\mathbb{D}$ :

$$
v(z):=\left(1-|z|^{2}\right)\left|f^{\prime}(z)\right| \exp \left(\left(1-|z|^{2}\right)\left|f^{\prime}(z)\right|\right)
$$


for $f(z)=(1+z) /(1-z)$. Easy calculations show that $\alpha_{\varphi}^{0}=\beta_{\varphi}^{0}=\alpha_{\varphi}^{\infty}=1$ but $\beta_{\varphi}^{\infty}=\infty$. On the other hand,

$$
-\Delta \log v(z)=\frac{1}{\left(1-|z|^{2}\right)^{2}},
$$

which implies that $L_{v}=U_{v}=1$.

5. Metric on $\mathbb{D}$ induced by a weight $v$ and stability of sequences of interpolation and sampling. We define $\delta_{z}(f):=f(z), z \in \mathbb{D}$, and

$$
\begin{aligned}
\varrho_{v}(z, p) & :=d\left(\frac{\delta_{z}}{\left\|\delta_{z}\right\|}, \operatorname{lin} \delta_{p}\right)=\inf \left\{\left\|\delta_{z} \widetilde{v}(z)-a \delta_{p}\right\|_{\left(B_{v}^{\infty}\right)^{\prime}}: a \in \mathbb{C}\right\} \\
& =\sup \left\{|f(z) \widetilde{v}(z)|:\|f\|_{v} \leq 1, f(p)=0\right\} .
\end{aligned}
$$

Since using $\varrho_{v}$ we can estimate from above the distance of $a$ to the unit circle, we easily obtain

$$
\varrho_{v}(z, p) \leq \inf _{|\lambda|=1}\left\|\frac{\delta_{z}}{\left\|\delta_{z}\right\|}-\frac{\lambda \delta_{p}}{\left\|\delta_{p}\right\|}\right\| \leq 2 \varrho_{v}(z, p)
$$

so $\varrho_{v}$ is equivalent to some metric on $\mathbb{D}$. Moreover, $\varrho_{v}=\varrho_{\widetilde{v}}$ and if $v \equiv 1$, then $\varrho_{v}=\varrho$.

Lemma 13. If $u=w / \widetilde{v}$ is an essential weight, then $C \varrho_{v} \leq \varrho_{w}$ for some constant $C>0$. In particular, for every weight $w$,

$$
\varrho_{w} \geq C \varrho \quad \text { for some constant } C>0 .
$$

Proof. Let $f(p)=0,\|f\|_{\tilde{v}} \leq 1$. For each $z \in \mathbb{D}$ there is a $g \in H(\mathbb{D})$ with $\|g\|_{u} \leq 1,|g(z)| \geq C \widetilde{v}(z) / w(z)$. Thus

$$
C|f(z) \widetilde{v}(z)| \leq|f(z) g(z) w(z)| \leq \varrho_{w}(z, p) .
$$

The following result is closely connected to [HKZ, Lemma 5.1] and generalizes [BP, Lemma] (cf. also [M, p. 395] and [Sch2, Lemma 4.2] for weighted Bergman spaces $\left.B_{v}^{p}, p<\infty\right)$.

Lemma 14. Let $v$ be a radial weight on $\mathbb{D}$ and let $f \in B_{v}^{\infty}$. Assume that there are $0<r<1$ and $C<\infty$ such that $v(z) / v(p) \leq C$ for all $z, p \in \mathbb{D}$ with $\varrho(z, p) \leq r$. Then

$$
|f(z)-f(p)| \leq \frac{4 C\|f\|_{v}}{r v(z)} \varrho(z, p)
$$

for all $z, p \in \mathbb{D}$ with $\varrho(z, p) \leq r / 2$.

Proof. Fix $p \in \mathbb{D}$. Since $\varphi_{p}\left(\varphi_{p}(z)\right)=z$ and $\varphi_{p}(0)=p$, we get

$$
|f(z)-f(p)|=\left|f\left(\varphi_{p}\left(\varphi_{p}(z)\right)\right)-f\left(\varphi_{p}(0)\right)\right| .
$$


For $|z|=\varrho\left(\varphi_{p}(z), p\right)=r$,

$$
\left|f\left(\varphi_{p}(z)\right)\right| \leq \frac{\|f\|_{v}}{v\left(\varphi_{p}(z)\right)} \leq \frac{\|f\|_{v}}{v(p)} \cdot \frac{v(p)}{v\left(\varphi_{p}(z)\right)} \leq \frac{C\|f\|_{v}}{v(p)} .
$$

Now consider $g_{p}:=f \circ \varphi_{p}$. Then, for $\varrho(z, p)=\left|\varphi_{p}(z)\right| \leq r / 2$,

$$
\begin{aligned}
|f(z)-f(p)| & =\left|g_{p}\left(\varphi_{p}(z)\right)-g_{p}(0)\right| \\
& \leq\left|g_{p}^{\prime}(\zeta)\right| \cdot\left|\varphi_{p}(z)\right|=\left|\varphi_{p}(z) \frac{1}{2 \pi} \int_{|\xi|=r} \frac{g_{p}(\xi)}{(\xi-\zeta)^{2}} d \xi\right|,
\end{aligned}
$$

since $|\zeta| \leq\left|\varphi_{p}(z)\right|<r / 2$. Thus

$$
|f(z)-f(p)| \leq\left|\varphi_{p}(z)\right| r \frac{C\|f\|_{v}}{\left(r-\left|\varphi_{p}(z)\right|\right)^{2}} \cdot \frac{1}{v(p)} \leq \frac{4 C\|f\|_{v}}{r v(p)} \varrho(z, p)
$$

for $\varrho(z, p) \leq r / 2$.

Corollary 15. If $v$ is an arbitrary essential weight with $U_{v}<\infty$, then $\varrho_{v} \sim \varrho$.

Proof. By Lemma 14, $\varrho_{p} \leq C_{p} \varrho$ for some constant $C_{p}>0$. Moreover, if $U_{v}<\infty$, then there is a $p>0$ such that

$$
-\Delta \log \frac{v_{p}}{v} \sim\left(1-|z|^{2}\right)^{-2}
$$

Apply Lemma 13 and Proposition 2(c).

The following result is known for weights $v$ satisfying $-\Delta \log v(z) \sim$ $\left(1-|z|^{2}\right)^{-2}$ (see [S3, Thm. 3], and for weights $v_{p}, p>0$, [BP, Thm. 9], [S2, Lemma 6.1]). For the multi-variable case see [M, Lemma 1.1].

COROLlary 16. Every set of interpolation for an arbitrary essential weight $v$ is $\varrho_{v}$-uniformly discrete. If $U_{v}<\infty$, then it is $\varrho$-uniformly discrete.

Proof. If $\left(z_{k}\right)$ is a set of interpolation for $v$, then there exist functions $f_{n} \in B_{v}^{\infty}$ and a constant $M>0$ such that $f_{n}\left(z_{n}\right) v\left(z_{n}\right)=1, f_{n}\left(z_{k}\right)=0$ if $k \neq n$, and $\left\|f_{n}\right\|_{v} \leq M$. Thus $\varrho_{v}\left(z_{n}, z_{k}\right)>1 / M$. Apply Corollary 15 .

It was shown in [Le, Sec. 6 II] (cf. [BP, Thm. 8], [JMT, Lemma 1.9]) that the sets of interpolation for $v_{p}$ are stable under small perturbations. To prove this for arbitrary weights $v$ we need the following lemma.

Lemma 17. Let $v$ be an arbitrary weight on $\mathbb{D}$. If $\left(z_{n}\right)$ is a sequence of distinct points in $\mathbb{D}$, then the following statements are equivalent:

(a) $\left(z_{n}\right)$ is a set of interpolation for $v$. 
(b) There is a constant $C>0$ such that

$$
\left\|\left(\xi_{n}\right)_{n}\right\|_{l_{1}} \leq C\left\|\sum_{n=1}^{\infty} \xi_{n} v\left(z_{n}\right) \delta_{z_{n}}\right\| \quad \text { for all }\left(\xi_{n}\right)_{n \in \mathbb{N}} \in l_{1} .
$$

If the unit ball of

$$
B_{v}^{0}:=\left\{f \in B_{v}^{\infty}: \lim _{|z| \rightarrow 1}|f(z)| v(z)=0\right\} \subseteq B_{v}^{\infty}
$$

is pointwise dense in some ball of $B_{v}^{\infty}$, then the above conditions are equivalent to

(c) The continuous linear map $R: B_{v}^{0}(\mathbb{D}) \rightarrow c_{0}, R(f)=\left(f\left(z_{n}\right) v\left(z_{n}\right)\right)_{n}$, is surjective, i.e. $\left(z_{n}\right)$ is a set of interpolation for $H_{v}^{0}(\mathbb{D})$.

Proof. Clearly, the unit ball $B_{v}$ of $B_{v}^{\infty}$ is compact in the compact-open topology co. Thus, by [BS, Thm. 1.1(a)], $\left(G_{v}^{\infty}\right)^{\prime}=B_{v}^{\infty}$, where

$$
G_{v}^{\infty}:=\left\{f \in\left(B_{v}^{\infty}\right)^{\prime}:\left.f\right|_{B_{v}} \text { is co-continuous }\right\} .
$$

Define a map $S: l_{1} \rightarrow G_{v}^{\infty}$ by

$$
S\left(\left(\xi_{n}\right)_{n}\right)=\sum_{n=1}^{\infty} \xi_{n} v\left(z_{n}\right) \delta_{z_{n}} .
$$

This is a well-defined continuous linear map. The adjoint map $S^{t}$ of $S$ coincides with the restriction operator $T$. Hence, $T$ is surjective if and only if (b) is fulfilled.

If the assumption of (c) holds, then by [BS, Thm. 1.1(b)], $G_{v}^{\infty}=\left(B_{v}^{0}\right)^{\prime}$ and $R^{t}=S$.

Lemma 18. Let $v$ be an essential weight on $\mathbb{D}$ and let $\left(z_{n}\right)$ be a set of interpolation (respectively a set of sampling) for $v$. Then there is a constant $0<\delta<1$ such that each sequence $\left(z_{n}^{\prime}\right)$ of distinct points in $\mathbb{D}$ satisfying $\varrho_{v}\left(z_{n}, z_{n}^{\prime}\right) \leq \delta$ for all $n$ is a set of interpolation (respectively of sampling) for $v$. If $U_{v}<\infty$ we can take @ instead of $\varrho_{v}$.

Proof. Assume that $\left(z_{n}\right)$ is a set of interpolation for $v$. By Lemma 17,

$$
\begin{aligned}
\left\|\left(\xi_{n}\right)_{n}\right\|_{l_{1}} & \leq C\left\|\sum_{n=1}^{\infty} \xi_{n} v\left(z_{n}\right) \delta_{z_{n}}\right\| \\
& \leq C\left(\left\|\sum_{n=1}^{\infty} \xi_{n} \lambda_{n} \widetilde{v}\left(z_{n}^{\prime}\right) \delta_{z_{n}^{\prime}}\right\|+\left\|\sum_{n=1}^{\infty} \xi_{n}\left(\lambda_{n} \widetilde{v}\left(z_{n}^{\prime}\right) \delta_{z_{n}^{\prime}}-\widetilde{v}\left(z_{n}\right) \delta_{z_{n}}\right)\right\|\right) \\
& \leq C\left(\left\|\sum_{n=1}^{\infty} \xi_{n} \lambda_{n} \widetilde{v}\left(z_{n}^{\prime}\right) \delta_{z_{n}^{\prime}}\right\|+2 \sup _{n \in \mathbb{N}} \varrho_{v}\left(z_{n}, z_{n}^{\prime}\right)\left\|\left(\xi_{n}\right)\right\|_{l_{1}}\right)
\end{aligned}
$$

for a suitably chosen sequence $\left(\lambda_{n}\right),\left|\lambda_{n}\right|=1$, not depending on $\left(\xi_{n}\right)_{n \in \mathbb{N}}$. This completes the proof of the first part by Lemma 17 . 
If $\left(z_{n}\right)$ is a set of sampling for $v$, then

$$
\begin{aligned}
\|f\|_{v} & \leq C \sup _{n \in \mathbb{N}}\left|f\left(z_{n}\right) v\left(z_{n}\right)\right| \\
& \leq C \sup _{n \in \mathbb{N}}\left|f\left(z_{n}^{\prime}\right) v\left(z_{n}^{\prime}\right)\right|+C\|f\|_{v} \sup _{n \in \mathbb{N}}\left\|\frac{\delta_{z_{n}}}{\left\|\delta_{z_{n}}\right\|}-\frac{\lambda_{n} \delta_{z_{n}^{\prime}}}{\left\|\delta_{z_{n}^{\prime}}\right\|}\right\| \\
& \leq C \sup _{n \in \mathbb{N}}\left|f\left(z_{n}^{\prime}\right) v\left(z_{n}^{\prime}\right)\right|+2 C\|f\|_{v} \sup _{n \in \mathbb{N}} \varrho_{v}\left(z_{n}, z_{n}^{\prime}\right) .
\end{aligned}
$$

By Corollary 15, $\varrho \sim \varrho_{v}$ if $U_{v}<\infty$.

COROLlary 19. If $v$ is an essential weight and $\left(z_{n}\right)$ is a set of sampling for $v$, then it contains a $\varrho_{v}$-uniformly discrete subsequence which is also a set of sampling for $v$. If $U_{v}<\infty$, we can take $\varrho$ instead of $\varrho_{v}$.

6. Comparison of weights. We start by making a simple but useful observation.

LEMMA 20. A sequence $\left(z_{n}\right) \subset \mathbb{D}$ is a set of linear interpolation for $v$ if and only if there is a sequence $\left(h_{n}\right) \subset B_{v}^{\infty}$ such that

$$
h_{n}\left(z_{k}\right)= \begin{cases}1 / v\left(z_{n}\right) & \text { if } k=n, \\ 0 & \text { if } k \neq n,\end{cases}
$$

and there is a constant $C$ such that

$$
\sum_{n=1}^{\infty}\left|h_{n}(z)\right| v(z) \leq C \quad \text { for every } z \in \mathbb{D} \text {. }
$$

Proof. We define a right inverse $R$ for $T$ by $R\left(\left(a_{n}\right)\right):=\sum_{n=1}^{\infty} a_{n} h_{n}$. This is clearly a well-defined, continuous map. On the other hand, if $R$ is an appropriate right inverse for $T$, then we take $h_{n}:=R\left(e_{n}\right) \in B_{v}^{\infty}$. Now easy duality arguments for $R_{\mid c_{0}}$ imply the existence of $C<\infty$ with $\sum_{n=1}^{\infty}\left|h_{n}(z)\right| v(z) \leq C$ for all $z \in \mathbb{D}$.

We apply the previous lemma to obtain the following comparison result (for other comparison type results see [JMT, Thm. 3.3], [M, Cor. 1.6], [S3, p. 720]).

Proposition 21. Let $v$ and $w$ be weights on $\mathbb{D}$ and let $\left(z_{n}\right) \subset \mathbb{D}$ be $a$ sequence.

(a) If $\left(z_{n}\right)$ is a set of interpolation for $v$ and there is a function $f \in B_{u}^{\infty}$, $u:=w / v$, such that $f\left(z_{n}\right) \geq 1 / u\left(z_{n}\right)$ for every $n \in \mathbb{N}$, then $\left(z_{n}\right)$ is a set of interpolation for $w$.

(b) If $\left(z_{n}\right)$ is a set of interpolation for $v$ and there is a sequence of functions $\left(g_{n}\right) \subset B_{u}^{\infty}, u:=w / v$, such that $g_{n}\left(z_{n}\right)=1 / u\left(z_{n}\right)$ for every 
$n \in \mathbb{N}$ and there is a constant $C<\infty$ with

$$
\sum_{n=1}^{\infty}\left|g_{n}(z)\right| u(z) \leq C \quad \text { for all } z \in \mathbb{D}
$$

then $\left(z_{n}\right)$ is a set of linear interpolation for $w$.

(c) If $\left(z_{n}\right)$ is a set of linear interpolation for $v$ and $u:=w / v$ is equivalent to an essential weight, then $\left(z_{n}\right)$ is a set of linear interpolation for $w$.

(d) If $\left(z_{n}\right)$ is a set of sampling for $v$ and $u:=v / w$ is equivalent to an essential weight, then $\left(z_{n}\right)$ is a set of sampling for $w$.

Note that the results above are not obvious because changing weights in interpolation or sampling we change not only the space but also the restriction map.

Proof. (a) Let $\left(a_{n}\right) \in l^{\infty}$ and let $b_{n}:=a_{n} /\left(f\left(z_{n}\right) u\left(z_{n}\right)\right)$. Clearly $\left(b_{n}\right) \in$ $l^{\infty}$. If $g \in B_{v}^{\infty}$ satisfies $g\left(z_{n}\right) v\left(z_{n}\right)=b_{n}$, then the function $h \in B_{w}^{\infty}, h(z):=$ $g(z) f(z)$, satisfies $h\left(z_{n}\right) w\left(z_{n}\right)=a_{n}$.

(b) Clearly, there is a sequence $\left(f_{n}\right)$ in $B_{v}^{\infty}$ such that

$$
f_{n}\left(z_{k}\right)= \begin{cases}1 / v\left(z_{n}\right) & \text { if } k=n \\ 0 & \text { if } k \neq n\end{cases}
$$

and, further, the Open Mapping Theorem yields a constant $M<\infty$ with $\left\|f_{n}\right\|_{v} \leq M$ for all $n \in \mathbb{N}$. Hence it is easily seen that the functions $h_{n}(z):=$ $f_{n}(z) g_{n}(z)$ in $B_{w}^{\infty}$ satisfy the conditions of Lemma 20.

(c) Since $u$ is equivalent to an essential weight, there is a bounded sequence $\left(f_{n}\right)$ in $B_{u}^{\infty}$ with $f_{n}\left(z_{n}\right)=1 / u\left(z_{n}\right)$. Moreover, there is a sequence $\left(g_{n}\right) \subset B_{v}^{\infty}$ satisfying the conditions of Lemma 20. Obviously, $\left(h_{n}\right) \subset B_{w}^{\infty}$ defined by $h_{n}(z):=f_{n}(z) g_{n}(z)$ satisfies the same conditions with $w$ in place of $v$ (with a different constant $C$ ).

(d) Assume that $\left(z_{n}\right)$ is not a set of sampling for $w$. Then for every $k \in \mathbb{N}$ there is a point $\xi_{k} \in \mathbb{D}$ and a function $f_{k} \in B_{w}^{\infty}$ such that $\left|f_{k}\left(\xi_{k}\right)\right| w\left(\xi_{k}\right) \geq k$ while $\sup _{n \in \mathbb{N}}\left|f_{k}\left(z_{n}\right)\right| w\left(z_{n}\right) \leq 1$. Now, there is a bounded sequence $\left(g_{k}\right)$ in $B_{u}^{\infty}$ such that $g_{k}\left(\xi_{k}\right)=1 / u\left(\xi_{k}\right)$. Clearly, $\left(h_{k}\right) \subset B_{v}^{\infty}, h_{k}(z):=g_{k}(z) f_{k}(z)$, and $\sup _{n \in \mathbb{N}}\left|h_{k}\left(z_{n}\right)\right| v\left(z_{n}\right) \leq\left\|g_{k}\right\|_{u} \leq C$ although $\left|h_{k}\left(\xi_{k}\right)\right| v\left(\xi_{k}\right) \geq k$. Thus $\left(z_{n}\right)$ cannot be a set of sampling for $v$.

Now, it makes sense to check which "weights" $u$ satisfy the corresponding conditions from Proposition 21.

LEMMA 22. For the weight $u(z)=\left(1-|z|^{2}\right)^{\varepsilon}, \varepsilon>0$, on $\mathbb{D}$ the assumptions of Proposition 21(b) are satisfied for each uniformly discrete sequence $\left(z_{n}\right)$. 
Proof. We define

$$
g_{n}(z):=\frac{1}{\left(1-\left|z_{n}\right|^{2}\right)^{\varepsilon}}\left(\frac{1-\left|z_{n}\right|^{2}}{1-\bar{z}_{n} z}\right)^{s}, \quad \text { where } s>1+\varepsilon .
$$

Thus $g_{n}\left(z_{n}\right)\left(1-\left|z_{n}\right|^{2}\right)^{\varepsilon}=1$ for all $n$. Further, for $z \in \mathbb{D}$ we have

$$
\sum_{n=1}^{\infty}\left|g_{n}(z)\right|\left(1-|z|^{2}\right)^{\varepsilon} \leq\left(1-|z|^{2}\right)^{\varepsilon} \sum_{n=1}^{\infty} \frac{\left(1-\left|z_{n}\right|^{2}\right)^{s-\varepsilon}}{\left|1-\bar{z}_{n} z\right|^{s}} .
$$

Since, by [S2, pp. 34-35], there is a constant $M>0$ such that for all $z \in \mathbb{D}$,

$$
\sum_{n=1}^{\infty} \frac{\left(1-\left|z_{n}\right|^{2}\right)^{s-\varepsilon}}{\left|1-\bar{z}_{n} z\right|^{s}} \leq M\left(1-|z|^{2}\right)^{-\varepsilon}
$$

whenever $\left(z_{n}\right)$ is uniformly discrete, the conclusion follows.

Now, we present some immediate consequences (for several variables a special case of the following result is also true; see [JMT, Thm. 3.3]).

Corollary 23. Let $v$ be an arbitrary essential weight. Then every set of interpolation for $H^{\infty}$ is also a set of linear interpolation for $v$. Analogously, every set of sampling for $B_{v}^{\infty}$ is also a set of sampling for $H^{\infty}$.

As the next application observe that Proposition 21(b) (together with Lemma 22 and Corollary 16) gives an elementary proof of the following result of Seip [S2] (for the unit ball in $\mathbb{C}^{n}$, see [M, Cor. 1.6]).

COROllary 24. Every set of interpolation for $v_{p}$ is a set of linear interpolation for $v_{q}$ for $0 \leq p<q$.

By Proposition 21(c) and 2(c) we obtain elementarily a much stronger result which under the additional assumption $-\Delta \log v(z) \sim\left(1-|z|^{2}\right)^{-2}$ also follows from [S3, Thm. 3].

COROLlaRY 25. If $v$ and $w$ are arbitrary smooth weights such that

$$
-\Delta \log \frac{w(z)}{v(z)} \sim\left(1-|z|^{2}\right)^{-2}
$$

then every set of linear interpolation for $v$ is also a set of linear interpolation for $w$.

Now, applying Proposition 21 we compare weights by means of our indices.

Corollary 26. Let $v, w$ be essential weights on $\mathbb{D}$.

(a) If $U_{w}<\infty$ and $L_{u}>0$ for $u:=w / v$, then every set of interpolation for $v$ is a set of linear interpolation for $w$.

(b) If $U_{v}<\infty$ and $L_{u}>0$ for $u:=v / w$, then every set of sampling for $v$ is a set of sampling for $w$. 
For radial weights $v$ and $w$ we have:

(c) If $U_{w}<\infty$ and $u:=w / v$ is almost decreasing, then every set of linear interpolation for $v$ is also a set of linear interpolation for $w$.

(d) If $U_{v}<\infty$ and $u:=v / w$ is almost decreasing, then every set of sampling for $v$ is also a set of sampling for $w$.

REMARK. If $U_{w}<\infty$ and $U_{v}<L_{w}$, then $L_{u}>0$ for $u:=w / v$ and the latter condition implies for radial weights that $u$ is almost decreasing (use Corollary 11 and the observation that $U_{u}<\infty$ ). This follows from the last part of the proof of Proposition 2(b) and some easy calculations.

Proof of Corollary 26. (a) First observe that $U_{v}<\infty$. Indeed,

$$
-\Delta \log v(z)=-\Delta \log w(z)+\Delta \log u(z) \leq \frac{U_{w}-L_{u}}{\left(1-|z|^{2}\right)^{2}} .
$$

Now, by Corollary 16, any set $\left(z_{n}\right)$ of interpolation for $v$ must be uniformly discrete. Hence, by Proposition 21(b) and Lemma 22, the sequence $\left(z_{n}\right)$ is a set of linear interpolation for the weight $v(z)(1-|z|)^{\varepsilon}, \varepsilon>0$. Choosing $\varepsilon<L_{u}$, we see that $L_{k}>0$ for $k(z):=w(z) /\left(v(z)(1-|z|)^{\varepsilon}\right)$. Consequently, by Propositions 2(c) and 21(c), it suffices to show that $U_{k}<\infty$, which follows easily from $U_{w}<\infty$.

(b) The proof is analogous.

(c) Since $U_{w}<\infty$, there are $q<\infty$ and $M$ such that $w(1-\lambda t) /\left(w(1-t) \lambda^{q}\right)$ $\geq M$ for all $\lambda, t \in(0,1]$. The weight $v$ is almost decreasing and we conclude that $U_{u}<\infty$. Consequently, Proposition 2(b) implies that $u$ is an essential weight. Now, the statement follows from Proposition 21(c).

(d) First, as above, one shows that $U_{u}<\infty$. Then, by Propositions 2(b) and $21(\mathrm{~d})$, the conclusion follows.

7. Main results. In order to get the final results we need two lemmas.

Lemma 27. For $r \in(1 / 2,1)$ and $\delta \in(0,1 / 2)$ we have the following inequalities:

(i) $\log \frac{1-\delta r}{r-\delta} \geq(1+\delta) \log \frac{1}{r}$;

(ii) $\log \frac{1+\delta r}{r+\delta} \leq(1-\delta) \log \frac{1}{r}$.

Proof. For $r=1$ both inequalities hold, so it suffices to check that for $\delta \in(0,1 / 2)$ the differences between both sides of the inequalities are suitably monotonic.

The following result is based on ideas contained in the proof of necessity of [S2, Thms. 1.1 and 1.2]. 
LEMMA 28. Let $\Gamma=\left(z_{n}\right)$ be a uniformly discrete sequence in $\mathbb{D}$ with $\varrho\left(z_{m}, z_{n}\right)>\beta$ for $m \neq n$ and $0<\delta<\beta<1 / 2$.

(a) If $D^{+}(\Gamma)<\infty$, then there is a sequence $\Gamma^{\prime}=\left(z_{n}^{\prime}\right) \subseteq \mathbb{D}$ with $\varrho\left(z_{n}, z_{n}^{\prime}\right) \leq \delta$ for $n \in \mathbb{N}$ such that

$$
D^{+}\left(\Gamma^{\prime}\right) \geq(1+\delta) D^{+}(\Gamma)
$$

(b) If $D^{-}(\Gamma)>0$, then there is a sequence $\Gamma^{\prime}=\left(z_{n}^{\prime}\right) \subseteq \mathbb{D}$ with $\varrho\left(z_{n}, z_{n}^{\prime}\right) \leq \delta$ for $n \in \mathbb{N}$ such that

$$
D^{-}\left(\Gamma^{\prime}\right) \leq(1-\delta) D^{-}(\Gamma)
$$

Proof. (a) Let $D^{+}(\Gamma)=\alpha$. Then there is a sequence $\left(w_{j}\right) \subseteq \mathbb{D}$ and an increasing sequence $\left(r_{j}\right)$ of positive numbers $r_{j} \rightarrow 1^{-}$such that

$$
D\left(\varphi_{w_{j}}(\Gamma), r_{j}\right) \geq \alpha-2^{-j}
$$

Let $S_{1}:=\left\{z \in \mathbb{D}: \varrho\left(z, \frac{1}{2} \mathbb{D}\right) \leq \beta\right\}$. Clearly $S_{1}$ is a Euclidean disc with center 0 and some radius $R$. Since for any $w \in \mathbb{D}, \varphi_{w}(\Gamma)$ is also $\beta$-discrete, there is a constant $K$ such that for any $w \in \mathbb{D}$ the set $S_{1}$ contains at most $K$ elements of $\varphi_{w}(\Gamma)$.

We split $\Gamma$ into disjoint finite sets as follows:

$$
\begin{aligned}
& \Gamma_{1}:=\left\{z_{k}: R<\left|\varphi_{w_{1}}\left(z_{k}\right)\right|<r_{1}\right\}, \\
& \Gamma_{j}:=\left\{z_{k}: R<\left|\varphi_{w_{j}}\left(z_{k}\right)\right|<r_{j}\right\} \backslash \bigcup_{l=1}^{j-1} \Gamma_{l} .
\end{aligned}
$$

Inductively, taking $r_{j}$ large enough, we may assume that

$$
\frac{\left(K+\operatorname{card} \bigcup_{l=1}^{j-1} \Gamma_{l}\right) \log 2}{\log \frac{1}{1-r_{j}}}<2^{-j}
$$

Finally,

$$
\left(\log \frac{1}{1-r_{j}}\right)^{-1} \sum_{z_{k} \in \Gamma_{j}} \log \frac{1}{\left|\varphi_{w_{j}}\left(z_{k}\right)\right|} \geq \alpha-2^{-j+1} \quad \text { for } j \in \mathbb{N} .
$$

We define

$$
z_{k}^{\prime}:=\varphi_{w_{j}}\left(\frac{\left|\varphi_{w_{j}}\left(z_{k}\right)\right|-\delta}{1-\delta\left|\varphi_{w_{j}}\left(z_{k}\right)\right|} \cdot \frac{\varphi_{w_{j}}\left(z_{k}\right)}{\left|\varphi_{w_{j}}\left(z_{k}\right)\right|}\right) \quad \text { for } z_{k} \in \Gamma_{j} .
$$

For $z_{k}$ belonging to no $\Gamma_{j}$ we define $z_{k}^{\prime}:=z_{k}$. It is known (see [Sh, Ex. 4.8.1]) that $\varphi_{w_{j}}\left(z_{k}^{\prime}\right)$ is the point at pseudohyperbolic distance $\delta$ from $\varphi_{w_{j}}\left(z_{k}\right)$ which has the smallest module.

By Lemma 27,

$$
\log \frac{1}{\left|\varphi_{w_{j}}\left(z_{k}^{\prime}\right)\right|} \geq(1+\delta) \log \frac{1}{\left|\varphi_{w_{j}}\left(z_{k}\right)\right|} \quad \text { for } z_{k} \in \Gamma_{j} .
$$


Hence

$$
\begin{aligned}
D\left(\varphi_{w_{j}}\left(\Gamma^{\prime}\right), r_{j}\right) & \geq(1+\delta)\left(\log \frac{1}{1-r_{j}}\right)^{-1} \sum_{z_{k} \in \Gamma_{j}} \log \frac{1}{\left|\varphi_{w_{j}}\left(z_{k}\right)\right|} \\
& \geq(1+\delta)\left(\alpha-2^{-j+1}\right) .
\end{aligned}
$$

Thus $\lim \sup _{r \rightarrow 1^{-}} \sup _{w \in \mathbb{D}} D\left(\varphi_{w}\left(\Gamma^{\prime}\right), r_{j}\right) \geq(1+\delta) \alpha$.

(b) Let $D^{-}(\Gamma)=\alpha$. Then there is a sequence $\left(w_{j}\right) \subseteq \mathbb{D}$ and a sequence $\left(r_{j}\right)$ of positive numbers $r_{j} \rightarrow 1^{-}$such that

$$
D\left(\varphi_{w_{j}}(\Gamma), r_{j}\right) \leq \alpha+2^{-j}
$$

We set $S_{j}:=\left\{z \in \mathbb{D}: \varrho\left(z, r_{j} \mathbb{D}\right) \leq \beta\right\}$. We split $\Gamma$ into disjoint finite sets as follows:

$$
\begin{aligned}
& \Gamma_{1}:=\left\{z_{k}: 1 / 2<\left|\varphi_{w_{1}}\left(z_{k}\right)\right|<r_{1}\right\}, \\
& \Gamma_{j}:=\left\{z_{k}: 1 / 2<\left|\varphi_{w_{j}}\left(z_{k}\right)\right|<r_{j}\right\} \backslash \bigcup_{l=1}^{j-1}\left\{z_{k}: \varphi_{w_{l}}\left(z_{k}\right) \subset S_{l}\right\} .
\end{aligned}
$$

Additionally, we define

$$
V_{j}:=\bigcup_{l=1}^{j-1}\left\{z_{k}: \varphi_{w_{l}}\left(z_{k}\right) \subset S_{l}\right\} .
$$

Inductively, taking $r_{j}$ large enough, we may assume that

$$
\frac{(\log 2) \operatorname{card} V_{j}}{\log \frac{1}{1-r_{j}}}<2^{-j} .
$$

We define

$$
z_{k}^{\prime}:=\varphi_{w_{j}}\left(\frac{\left|\varphi_{w_{j}}\left(z_{k}\right)\right|+\delta}{1+\delta\left|\varphi_{w_{j}}\left(z_{k}\right)\right|} \cdot \frac{\varphi_{w_{j}}\left(z_{k}\right)}{\left|\varphi_{w_{j}}\left(z_{k}\right)\right|}\right) \quad \text { for } z_{k} \in \Gamma_{j} .
$$

For $z_{k}$ belonging to no $\Gamma_{j}$ we define $z_{k}^{\prime}:=z_{k}$. It is known (see [Sh, Ex. 4.8.1]) that $\varphi_{w_{j}}\left(z_{k}^{\prime}\right)$ is the point at pseudohyperbolic distance $\delta$ from $\varphi_{w_{j}}\left(z_{k}\right)$ which has the greatest module.

By Lemma 27,

$$
\log \frac{1}{\left|\varphi_{w_{j}}\left(z_{k}^{\prime}\right)\right|} \leq(1-\delta) \log \frac{1}{\left|\varphi_{w_{j}}\left(z_{k}\right)\right|} \quad \text { for } z_{k} \in \Gamma_{j} .
$$

Hence,

$$
\begin{aligned}
& D\left(\varphi_{w_{j}}\left(\Gamma^{\prime}\right), r_{j}\right) \\
& \quad \leq\left(\log \frac{1}{1-r_{j}}\right)^{-1}\left(\sum_{z_{k} \in \Gamma_{j}} \log \frac{1}{\left|\varphi_{w_{j}}\left(z_{k}^{\prime}\right)\right|}+\sum_{z_{k} \in V_{j}} \log \frac{1}{\left|\varphi_{w_{j}}\left(z_{k}^{\prime}\right)\right|}\right)
\end{aligned}
$$




$$
\begin{aligned}
& \leq\left(\log \frac{1}{1-r_{j}}\right)^{-1}(1-\delta) \sum_{z_{k} \in \Gamma_{j}} \frac{1}{\left|\varphi_{w_{j}}\left(z_{k}\right)\right|}+2^{-j} \\
& \leq(1-\delta)\left(\alpha+2^{-j}\right)+2^{-j} .
\end{aligned}
$$

Therefore, $D^{-}\left(\Gamma^{\prime}\right) \leq(1-\delta) \alpha$.

By the results of Seip [S2] and Corollary 26 we now obtain the following result.

Theorem 29. Let $v$ be an essential weight on $\mathbb{D}$ such that $U_{v}<\infty$.

(a) Every uniformly discrete sequence $\Gamma=\left(z_{n}\right)$ of density $D^{+}(\Gamma)<L_{v}$ is a set of linear interpolation for $v$.

(b) If $\Gamma=\left(z_{n}\right)$ is a set of interpolation for $v$, then $\Gamma$ is uniformly discrete and $D^{+}(\Gamma)<U_{v}$ or $D^{+}(\Gamma)=0$ if $U_{v}=0$.

(c) If the sequence $\Gamma=\left(z_{n}\right)$ contains a uniformly discrete subset $\Gamma^{\prime}$ of density $D^{-}\left(\Gamma^{\prime}\right)>U_{v}$, then $\Gamma$ is a set of sampling for $v$.

(d) If $L_{v}>0$ and $\Gamma=\left(z_{n}\right)$ is a set of sampling for $v$, then $\Gamma$ contains a uniformly discrete subset $\Gamma^{\prime}$ with $D^{-}\left(\Gamma^{\prime}\right)>L_{v}$.

Proof. (a) Let $0<\varepsilon<L_{v}-D^{+}(\Gamma)$, so $L_{v}-\varepsilon>D^{+}(\Gamma)$. Since $\Gamma$ is uniformly discrete, Theorem B implies that $\Gamma$ is a set of interpolation for the weight $w(z)=\left(1-|z|^{2}\right)^{L_{v}-\varepsilon}$. Now, $U_{w}=L_{v}-\varepsilon<L_{v}$, so Corollary 26(a) and Remark after it show that $\Gamma$ is a set of linear interpolation for $v$.

(b) By Corollary 16, $\Gamma$ is uniformly discrete. Take an arbitrary $\varepsilon>0$. Then $U_{v}+\varepsilon<\infty$ and for $w(z)=\left(1-|z|^{2}\right)^{U_{v}+\varepsilon}$ it follows that $U_{v}<U_{v}+\varepsilon=$ $L_{w}$. Hence $\Gamma$ is a set of linear interpolation for $w$ by Corollary 26(a). By Theorem B, $D^{+}(\Gamma)<U_{v}+\varepsilon$ for every $\varepsilon>0$. Thus $D^{+}(\Gamma) \leq U_{v}$.

By Lemma 18, we choose $\delta$ for $\Gamma$ as a set of interpolation for $v$. Take a sequence $\Gamma^{\prime}=\left(z_{n}^{\prime}\right)$ in $\mathbb{D}$ such that $\varrho\left(z_{n}, z_{n}^{\prime}\right) \leq \delta$ for all $n$ and $(1+\delta) D^{+}(\Gamma) \leq$ $D^{+}\left(\Gamma^{\prime}\right)$ (use Lemma 28). By Lemma 18, the set $\Gamma^{\prime}$ is a set of interpolation for $v$, so the above argument shows that $D^{+}\left(\Gamma^{\prime}\right) \leq U_{v}$. Hence $D^{+}(\Gamma)<U_{v}$.

(c) Let $0<\varepsilon<D^{-}(\Gamma)-U_{v}$. Thus, by Theorem A, the set $\Gamma$ is a set of sampling for $w(z)=\left(1-|z|^{2}\right)^{U_{v}+\varepsilon}$. Since $U_{w}=U_{v}+\varepsilon<\infty$ and $L_{w}=U_{v}+\varepsilon>U_{v}$, Corollary 26(b) gives the statement.

(d) By Corollary 19, we may assume that $\Gamma=\left(z_{k}\right)$ is uniformly discrete where

$$
\varrho\left(z_{m}, z_{n}\right) \geq \beta \quad \text { for } m \neq n .
$$

Take an arbitrary $0<\varepsilon<1$. Then $U_{w}=\varepsilon L_{v}<L_{v}$ for the weight $w(z)=$ $\left(1-|z|^{2}\right)^{\varepsilon L_{v}}$. Hence $\Gamma$ is a set of sampling for $w$ by Corollary 26(b). Since Theorem A yields $D^{-}(\Gamma)>\varepsilon L_{v}$ for any $\varepsilon$, we have $D^{-}(\Gamma) \geq L_{v}$.

By Lemma 18, we choose $\delta$ for $\Gamma$ as a set of sampling for $v$. We may assume that $\delta<\beta / 3$. By Lemma 28, we choose a sequence $\Gamma^{\prime}=\left(z_{k}^{\prime}\right)$ in $\mathbb{D}$ such that $\varrho\left(z_{n}^{\prime}, z_{n}\right) \leq \delta$ and $D^{-}\left(\Gamma^{\prime}\right) \leq(1-\delta) D^{-}(\Gamma)$. By Lemma 18, the 
set $\Gamma^{\prime}$ is a set of sampling for $v$, and it is uniformly discrete. Thus, by the above arguments, $D^{-}\left(\Gamma^{\prime}\right) \geq L_{v}$ and

$$
L_{v} \leq D^{-}\left(\Gamma^{\prime}\right) \leq(1-\delta) D^{-}(\Gamma) .
$$

Corollary 30. Let $v$ be an essential weight on $\mathbb{D}$ and $U_{v}=0$. Then every set of interpolation $\Gamma$ for $v$ is uniformly discrete and $D^{+}(\Gamma)=0$.

The above corollary applies for instance to any weight $v$ which is defined by

$$
v(z)=\left(\log \left(\frac{e}{1-|z|}\right)\right)^{\varepsilon}
$$

where $\varepsilon<0$, or any logarithmic weight as in [SW1, p. 265]. These weights are not covered by Seip's theory [S3] because $-\Delta \log v(z)=o\left(\left(1-|z|^{2}\right)^{-2}\right)$.

Corollary 31. Let $v$ be an essential weight on $\mathbb{D}$ such that $0<L_{v}=$ $U_{v}<\infty$.

(a) $\Gamma$ is a set of interpolation for $v$ if and only if $\Gamma$ is uniformly discrete and $D^{+}(\Gamma)<U_{v}$.

(c) $\Gamma$ is a set of sampling for $v$ if and only if $\Gamma$ contains a uniformly discrete subset $\Gamma^{\prime}$ with $D^{-}\left(\Gamma^{\prime}\right)>L_{v}$.

The above corollary means that if $U_{v}=L_{v}=p$ then the spaces $B_{v}^{\infty}$ and $B_{v_{p}}^{\infty}$ have exactly the same sets of interpolation and sets of sampling, although they can be essentially different. As one easily calculates this holds, for instance, for

$$
v(z)=\left(1-|z|^{2}\right)^{p}\left(\log \left(\frac{e}{1-|z|}\right)\right)^{\varepsilon}
$$

where $\varepsilon \in \mathbb{R}$, or even

$$
v(z)=\left(\left(1-|z|^{2}\right)\left|f^{\prime}(z)\right|\right)^{p}\left(\log \left(\frac{e}{\left(1-|z|^{2}\right)\left|f^{\prime}(z)\right|}\right)\right)^{\varepsilon},
$$

where $f: \mathbb{D} \rightarrow \Omega$ is an arbitrary Riemann map.

The possibility of such a phenomenon can also be deduced from the results in [S3], but our machinery allows us to identify easily such weights. Using Corollaries 11 and 12 we may formulate the following result.

Corollary 32. Let $\varphi: \mathbb{R}_{+} \rightarrow \mathbb{R}^{+}$be an almost increasing function with $0<\alpha_{\varphi}^{\infty}=\beta_{\varphi}^{\infty}<\infty$ (for instance, $\left.\varphi(t)=t^{p}\right)$. Let $\Omega$ be an arbitrary simply connected domain and $g: \Omega \rightarrow \mathbb{D}$ be a Riemann map. Let $v: \Omega \rightarrow \mathbb{R}_{+}$, $v(z)=\varphi(\operatorname{dist}(z, \partial \Omega))$.

(a) $A$ set $\Gamma \subseteq \Omega$ is a set of interpolation for $B_{v}^{\infty}(\Omega)$ if and only if $g(\Gamma)$ is uniformly discrete and $D^{+}(g(\Gamma))<\alpha_{\varphi}^{\infty}$. 
(b) A set $\Gamma \subseteq \Omega$ is a set of sampling for $B_{v}^{\infty}(\Omega)$ if and only if $\Gamma$ contains a subset $\Gamma^{\prime}$ such that $g\left(\Gamma^{\prime}\right)$ is uniformly discrete and $D^{-}\left(g\left(\Gamma^{\prime}\right)\right)>\alpha_{\varphi}^{\infty}$.

If $\Omega$ is thin, then we have the same result for $\alpha_{\varphi}^{0}, \beta_{\varphi}^{0}$ in place of $\alpha_{\varphi}^{\infty}, \beta_{\varphi}^{\infty}$.

Let us mention that on the strip $\Omega:=\{z:|\operatorname{Im} z|<\pi / 2\}$ the Riemann map $g: \Omega \rightarrow \mathbb{D}$ is given by the formula

$$
g(z):=\frac{e^{z}-1}{e^{z}+1}
$$

A sequence $\Gamma \subseteq \Omega$ is a set of interpolation for $B_{v}^{\infty}, v(z)=\cos ^{2 p}(\operatorname{Im} z)$, $p>0$, if and only if $\Gamma$ is uniformly discrete and

$$
D^{+}(g(\Gamma))<p .
$$

Acknowledgements. The authors are greatly indebted to L. Frerick (Wuppertal), A. Michalak (Poznań) and K. Seip (Trondheim) for discussions on the subject of the paper.

\section{References}

[AD] J. M. Anderson and J. Duncan, Duals of Banach spaces of entire functions, Glasgow Math. J. 32 (1990), 215-220.

[B] B. Berndtsson, Interpolating sequences for $H^{\infty}$ in the ball, Indag. Math. 88 (1985), 1-11.

[BO] B. Berndtsson and J. Ortega Cerdà, On interpolation and sampling in Hilbert spaces of analytic functions, J. Reine Angew. Math. 464 (1995), 109-128.

[BBT] K. D. Bierstedt, J. Bonet and J. Taskinen, Associated weights and spaces of holomorphic functions, Studia Math. 127 (1998), 137-168.

[BS] K. D. Bierstedt and W. H. Summers, Biduals of weighted Banach spaces of analytic functions, J. Austral. Math. Soc. (Series A) 54 (1993), 70-79.

[BDL1] J. Bonet, P. Domański and M. Lindström, Essential norm and weak compactness of composition operators on weighted Banach spaces of analytic functions, Canad. Math. Bull. 42 (1999), 139-148.

[BDL2] - - - - Pointwise multiplication operators on weighted Banach spaces of analytic functions, Studia Math. 137 (1999), 177-194.

[BDLT] J. Bonet, P. Domański, M. Lindström and J. Taskinen, Composition operators between weighted Banach spaces of analytic functions, J. Austral. Math. Soc. Ser. A 64 (1998), 101-118.

[BP] J. Bruna and D. Pascuas, Interpolation in $A^{-\infty}$, J. London Math. Soc. 40 (1989), 452-466.

[G] J. B. Garnett, Bounded Analytic Functions, Academic Press, 1981.

[GW] K. Gröchenig and D. Walnut, A Riesz basis for Bargmann-Fock space related to sampling and interpolation, Ark. Mat. 30 (1992), 283-295.

[HKZ] H. Hedenmalm, B. Korenblum and K. Zhu, Theory of Bergman Spaces, Springer, New York, 2000. 
[JMT] M. Jevtić, X. Massaneda and P. J. Thomas, Interpolating sequences for weighted Bergman spaces of the ball, Michigan Math. J. 43 (1996), 495-517.

[K] B. Korenblum, An extension of the Nevanlinna theory, Acta Math. 135 (1975), 187-219.

[LT] J. Lindenstrauss and L. Tzafriri, Classical Banach Spaces, Springer, Berlin, 1977.

[Le] D. Luecking, Forward and reverse Carleson inequalities for functions in Bergman spaces and their derivatives, Amer. J. Math. 107 (1985), 85-111.

[L1] W. Lusky, On the structure of $H v_{0}(D)$ and $h v_{0}(D)$, Math. Nachr. 159 (1992), 279-289.

[L2] - On weighted spaces of harmonic and holomorphic functions, J. London Math. Soc. 51 (1995), 309-320.

[LS] Y. I. Lyubarskiı̌ and K. Seip, Sampling and interpolation of entire functions and exponential systems in complex domains, Ark. Mat. 32 (1994), 157-193.

[Mal] L. Maligranda, Indices and interpolation, Dissertationes Math. 234 (1985).

[M] X. Massaneda, $A^{-p}$ interpolation in the unit ball, J. London Math. Soc. 52 (1995), 391-401.

[Po] C. Pommerenke, Boundary Behaviour of Conformal Maps, Springer, Berlin, 1992.

[RS] L. A. Rubel and A. L. Shields, The second duals of certain spaces of analytic functions, J. Austral. Math. Soc. 11 (1970), 276-280.

[Sch1] A. P. Schuster, Sets of sampling and interpolation in Bergman spaces, Proc. Amer. Math. Soc. 125 (1997), 1717-1725.

[Sch2] - On Seip's theorems for sampling and interpolation in Bergman spaces, preprint.

[ScS1] A. P. Schuster and K. Seip, A Carleson-type condition for interpolation in Bergman spaces, J. Reine Angew. Math. 497 (1998), 223-233.

[ScS2] - - - Weak conditions for interpolation in holomorphic spaces, Publ. Mat. 44 (2000), 277-293.

[S1] K. Seip, Density theorems for sampling and interpolation in the Bargmann-Fock space, Bull. Amer. Math. Soc. 26 (1992), 322-328.

[S2] - Beurling type density theorems in the unit disc, Invent. Math. 113 (1993), 21-39.

[S3] - Developments from nonharmonic Fourier series, Doc. Math. 1988, Extra Vol. II (ICM 1998), 713-722.

[S4] - On Korenblum's density condition for the zero sequences of $A^{-\alpha}$, J. Anal. Math. 67 (1995), 307-322.

[Sh] J. H. Shapiro, Composition Operators and Classical Function Theory, Springer, Berlin, 1993.

[SW1] A. L. Shields and D. L. Williams, Bounded projections, duality, and multipliers in spaces of harmonic functions, J. Reine Angew. Math. 299/300 (1978), 256279.

[SW2] - - - Bounded projections and the growth of harmonic conjugates in the disc, Michigan Math. J. 29 (1982), 3-25.

[Sd] S. V. Shvedenko, Hardy classes and related spaces of analytic functions on the unit disc, polydisc and ball, Itogi Nauki i Tekhniki, Mat. Anal. 23 (1985), 3-124 (in Russian).

[T] J. Taskinen, Compact composition operators on general weighted spaces, Houston J. Math. 27 (2001), 203-218. 
[W] P. Wojtaszczyk, Banach Spaces for Analysts, Cambridge Univ. Press, Cambridge, 1991.

[Z1] K. Zhu, Operator Theory in Function Spaces, Dekker, New York, 1990.

[Z2] - Evaluation operator on the Bergman space, Math. Proc. Cambridge Philos. Soc. 117 (1995), 513-523.

Faculty of Mathematics and Computer Science

A. Mickiewicz University

and

Institute of Mathematics

Polish Academy of Sciences

Poznań Branch

Umultowska 87

61-614 Poznań, Poland

E-mail: domanski@amu.edu.pl
Department of Mathematics Åbo Akademi University FIN-20500 Åbo, Finland E-mail: mikael.lindstrom@abo.fi 